\title{
Take Off And Landing Stage Of Aircraft with Matlab Simulation
}

\author{
Er. Naser.F.AB.Elmajdub1, Dr.A.K. Bharadwaj2 \\ 1,2 (Department, of Electrical Engineering, SHIATS Allahabad, India)
}

\begin{abstract}
The simulation contains a comprehensive view about the stage of takeoff and landing with auto control. This model display the simulation of takeoff and landing of aircraft with the help of simulink matlab. Some useful graph explain about positions angles and altitude of aircraft. In this model auto controller are used. Starting and journey of the model by the different Engineers . Mr Naser Give GUI based simulation work in his sereach study in Phd.This model run on matlab software. In matlab many block for design the aircraft take off and landing stages.
\end{abstract}

\section{Introduction}

This model is a variant of the vrtkoff example that shows how to trace trajectory of a moving object (the plane) in the scene.VR Tracer block adds the following behavior to the scene visualization:A marker is placed at the current position of the traced object in regular time intervals specified by the block sample time. Markers form a visible trace of the object's trajectory.

The distance between markers indicates the speed of the object at given position.In addition, marker color can be dynamically set using the second block input to represent another model property or status.

VR Tracer block allows you to specify the following parameters:

- Associated VRML File

- Parent node of markers to create (useful when the traced object is inside other object in the scene hierarchy)

- General marker shape to be selected from the listbox (select None for displaying line / triads below only)

- Checkboxes to select whether you want to display trajectory as a line and/or axis-aligned triads

- Marker scale

- Marker color, to be defined in block input or in the block mask.

- Sample time

- Checkbox to ensure that a viewer window is open during simulation

In this example, a tetrahedron shape is used as a general shape marker, together with both trajectory line and triads.

\section{Review And Letretural}

Aircraft take off and landing stages were designed in 2007. Mr Naser was reseach more in takeoff and landing and give some improved design using matlab simulation. In 2009 Mr. Grijesh Singh worked for landing of aircraft and give great job in simulation but some problem in autocrollers. Same time Mr Jaccob Itlacy give take off and landing program with using $C$ Language and apreceated by all, great job.

Mr. Naser continuously reseach for takeoff and landing using matlab simulation. In $2012 \mathrm{Mr}$ naser again made some simulation of landing in his M Tech Program.Finally in 2014 Mr Naser give both take off and landing stage using GUI program in matlab. In matlab he give the great achievement and thanks to matlab software group.

Design Control Take Off Stage A/C

\section{Methedology}

\section{Plane Take-Off with Trajectory Tracing}

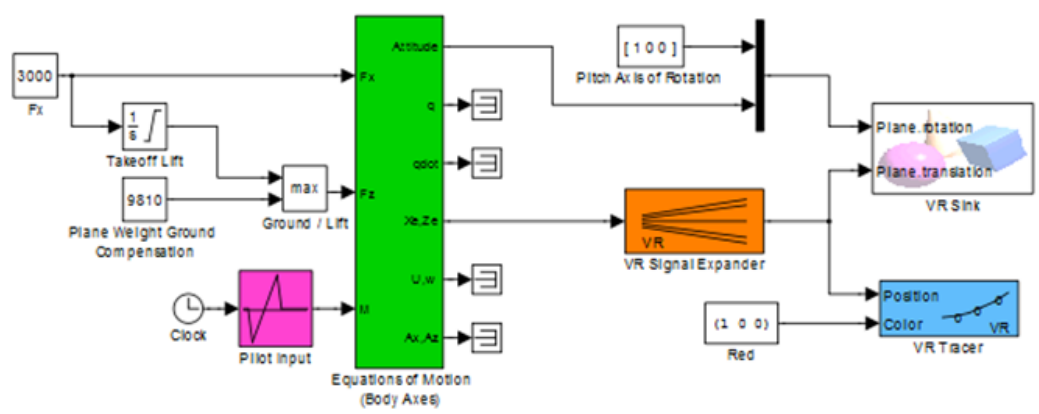


Full Model Hierarchy

1. take off

\begin{tabular}{|l|l|}
\hline Simulation Parameter & Value \\
\hline Solver & ode45 \\
\hline RelTol & $1 \mathrm{e}-3$ \\
\hline Refine & 1 \\
\hline MaxOrder & 5 \\
\hline ZeroCross & on \\
\hline
\end{tabular}

Table 1. 3 DOF equations of motion Block Properties

\begin{tabular}{|l|l|l|l|l|l|l|l|l|}
\hline Name & V ini & Theta ini & Alpha ini & Q ini & Pos ini & Mass & Iyy & G \\
\hline Equations of Motion (Body Axes) & 0 & 0 & 0 & 0 & {$[00]$} & 1000 & 1000 & -9.81 \\
\hline
\end{tabular}

Table 2. Clock Block Properties

\begin{tabular}{|l|l|l|}
\hline Name & Display Time & Decimation \\
\hline Clock & off & 10 \\
\hline
\end{tabular}

Table 3. Constant Block Properties

\begin{tabular}{|c|c|c|c|c|c|c|c|}
\hline Name & Value & Sampling Mode & $\begin{array}{l}\text { Out } \\
\text { Min }\end{array}$ & Out Max & Out Data Type Str & Sample Time & $\begin{array}{l}\text { Frame } \\
\text { Period }\end{array}$ \\
\hline Fx & 3000 & Sample based & [] & [] & $\begin{array}{l}\text { Inherit: Inherit from } \\
\text { 'Constant value' }\end{array}$ & inf & inf \\
\hline Pitch Axis of Rotation & {$\left[\begin{array}{lll}1 & 0 & 0\end{array}\right]$} & Sample based & [] & [] & $\begin{array}{l}\text { Inherit: Inherit from } \\
\text { 'Constant value' }\end{array}$ & inf & inf \\
\hline $\begin{array}{l}\text { Plane Weight } \quad \text { Ground } \\
\text { Compensation }\end{array}$ & 9810 & Sample based & [] & [] & \begin{tabular}{|l|} 
Inherit: Inherit from \\
'Constant value'
\end{tabular} & inf & inf \\
\hline Red & {$\left[\begin{array}{lll}1 & 0 & 0\end{array}\right]$} & Sample based & [] & [] & $\begin{array}{l}\text { Inherit: Inherit from } \\
\text { 'Constant value' }\end{array}$ & inf & inf \\
\hline
\end{tabular}

Table 4. Integrator Block Properties

\begin{tabular}{|c|c|c|c|c|c|c|c|c|c|c|}
\hline Name & External Reset & $\begin{array}{l}\text { Initial } \\
\text { Source }\end{array}$ & Condition & $\begin{array}{l}\text { Limit } \\
\text { Output }\end{array}$ & $\begin{array}{l}\text { Upper } \\
\text { Limit }\end{array}$ & Saturation & $\begin{array}{l}\text { Lower } \\
\text { Limit }\end{array}$ & Saturation & \begin{tabular}{|l} 
Zero \\
Cross
\end{tabular} & $\begin{array}{l}\text { Continuous State } \\
\text { Attributes }\end{array}$ \\
\hline Takeoff Lift & none & internal & & on & 11000 & & -Inf & & off & $"$ \\
\hline
\end{tabular}

Table 5. Lookup Block Properties

\begin{tabular}{|c|c|c|c|c|c|c|}
\hline Name & Input Values & Table & Look Up Meth & Out Min & Out Max & Out Data Type Str \\
\hline Pilot Input & {$\left[\begin{array}{llllll}0 & 1 & 2 & 8 & 9 & 15\end{array}\right]$} & 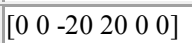 & Interpolation-Extrapolation & [] & [] & Inherit: Same as input \\
\hline
\end{tabular}

Table 6. MinMax Block Properties

\begin{tabular}{|l|l|l|l|l|l|l|l|}
\hline Name & Function & Inputs & Input Same DT & Out Min & Out Max & Out Data Type Str & Zero Cross \\
\hline Ground / Lift & $\max$ & 2 & on & {[]} & {[]} & Inherit: Inherit via internal rule & off \\
\hline
\end{tabular}

Table 7. Mux Block Properties

\begin{tabular}{|l|l|l|}
\hline Name & Inputs & Display Option \\
\hline Mux3 & 2 & bar \\
\hline
\end{tabular}

Table 8. Terminator Block Properties

\begin{tabular}{|l|}
\hline Name \\
\hline Terminator1 \\
\hline Terminator2 \\
\hline Terminator3 \\
\hline Terminator6 \\
\hline
\end{tabular}

Table 9. VR Signal Expander Block Properties

\begin{tabular}{|l|l|l|}
\hline Name & Outwidth & Outidx \\
\hline VR Signal Expander & 3 & {$\left[\begin{array}{ll}3 & 2\end{array}\right]$} \\
\hline
\end{tabular}


Take Off And Landing Stage Of Aircraft with Matlab Simulation

Table 10. VR Tracer Block Properties

\begin{tabular}{|c|c|c|c|c|c|c|c|c|c|}
\hline Name & Vrml File & Marker Name & Use Lines & Use Triads & Marker Scale & $\begin{array}{l}\text { Marker Color } \\
\text { Selection }\end{array}$ & Ts & $\begin{array}{l}\text { Force } \\
\text { Open }\end{array}$ & Viewer \\
\hline VR Tracer & vrtkoff.wrl & Tetrahedron & on & on & {$\left[\begin{array}{lll}1 & 1 & 1\end{array}\right]$} & Block input & 0.5 & off & \\
\hline
\end{tabular}

Table 11. Virtual Reality Sink Block Properties

\begin{tabular}{|l|l|l|l|l|l|l|l|l|l|l|l|l|l|}
\hline Name & $\begin{array}{l}\text { Sample } \\
\text { Time }\end{array}$ & $\begin{array}{l}\text { View } \\
\text { Enable }\end{array}$ & $\begin{array}{l}\text { Remote } \\
\text { View }\end{array}$ & Fields Written & $\begin{array}{l}\text { World File } \\
\text { Name }\end{array}$ & $\begin{array}{l}\text { World } \\
\text { Description }\end{array}$ & $\begin{array}{l}\text { Auto } \\
\text { View }\end{array}$ & $\begin{array}{l}\text { Video } \\
\text { Dimensions }\end{array}$ \\
\hline $\begin{array}{l}\text { VR } \\
\text { Sink }\end{array}$ & 0.05 & on & off & Plane.rotation.4.1.double\#Plane.translation.3.1.double & vrtkoff.wrl & $\begin{array}{l}\text { VR } \\
\text { Take-Off }\end{array}$ \\
\hline
\end{tabular}

Table 12. Block Type Count

\begin{tabular}{|l|l|l|}
\hline BlockType & Count & Block Names \\
\hline Terminator & 4 & $\underline{\text { Terminator1, Terminator2, Terminator3, Terminator6 }}$ \\
\hline Constant & 4 & $\underline{\text { Fx }, \text { Pitch Axis of Rotation, Plane Weight Ground Compensation, Red }}$ \\
\hline Virtual Reality Sink $(\mathrm{m})$ & 1 & VR Sink \\
\hline VR Tracer $(\mathrm{m})$ & 1 & VR Tracer \\
\hline VR Signal Expander $(\mathrm{m})$ & 1 & VR Signal Expander \\
\hline Mux & 1 & Mux3 \\
\hline MinMax & 1 & Ground / Lift \\
\hline Lookup & 1 & Pilot Input \\
\hline Integrator & 1 & Takeoff Lift \\
\hline Clock & 1 & Clock \\
\hline 3 DOF equations of motion $(\mathrm{m})$ & 1 & Equations of Motion (Body Axes) \\
\hline
\end{tabular}

Table 13. Model Functions

\begin{tabular}{|l|l|l|}
\hline Function Name & Parent Blocks & Calling string \\
\hline Inf & Takeoff Lift & -Inf \\
\hline
\end{tabular}

\section{Result of Simulation of Take Off}

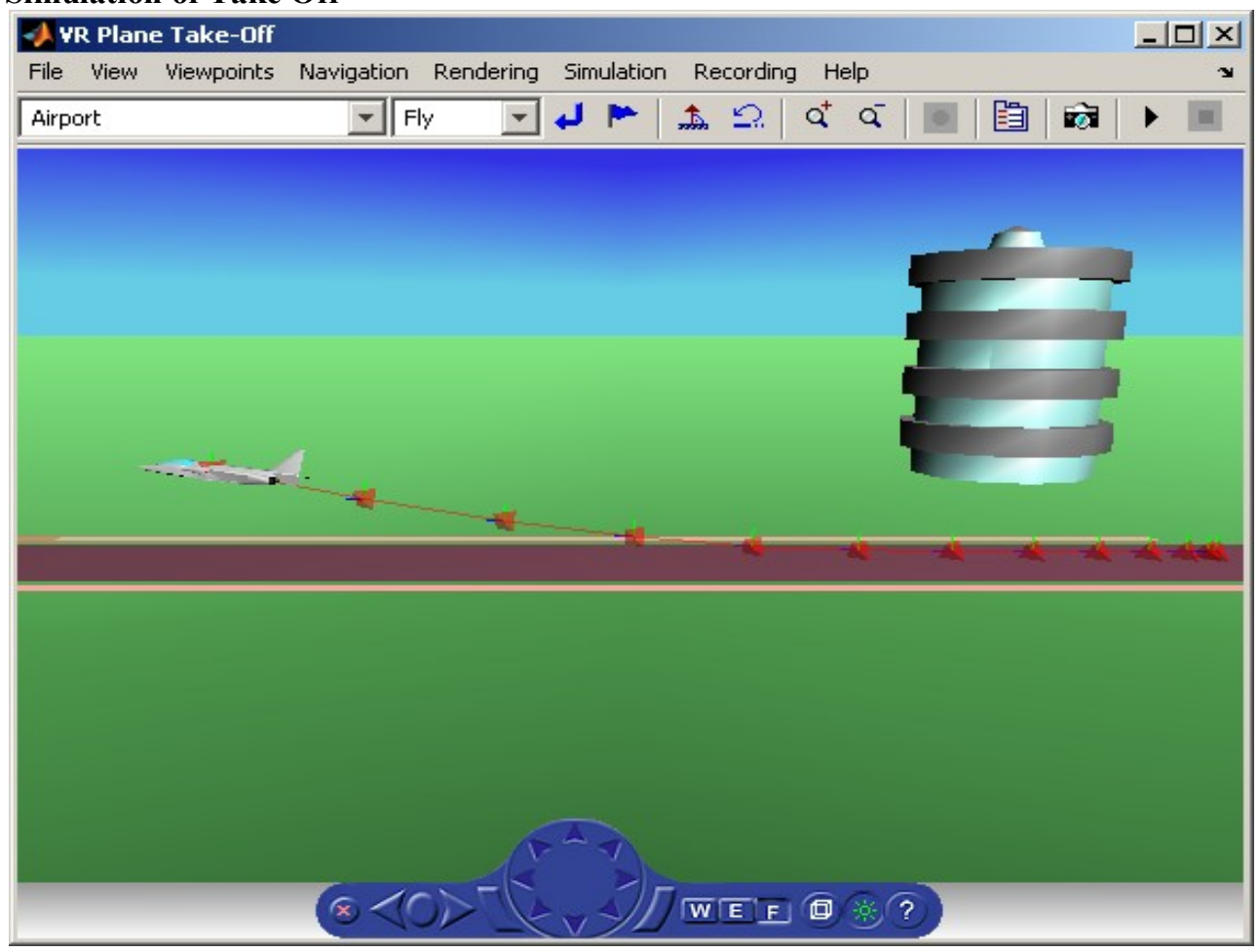




\section{Landing Stage Of Aircraft}

\section{Landing of Aircraft with Controllers}

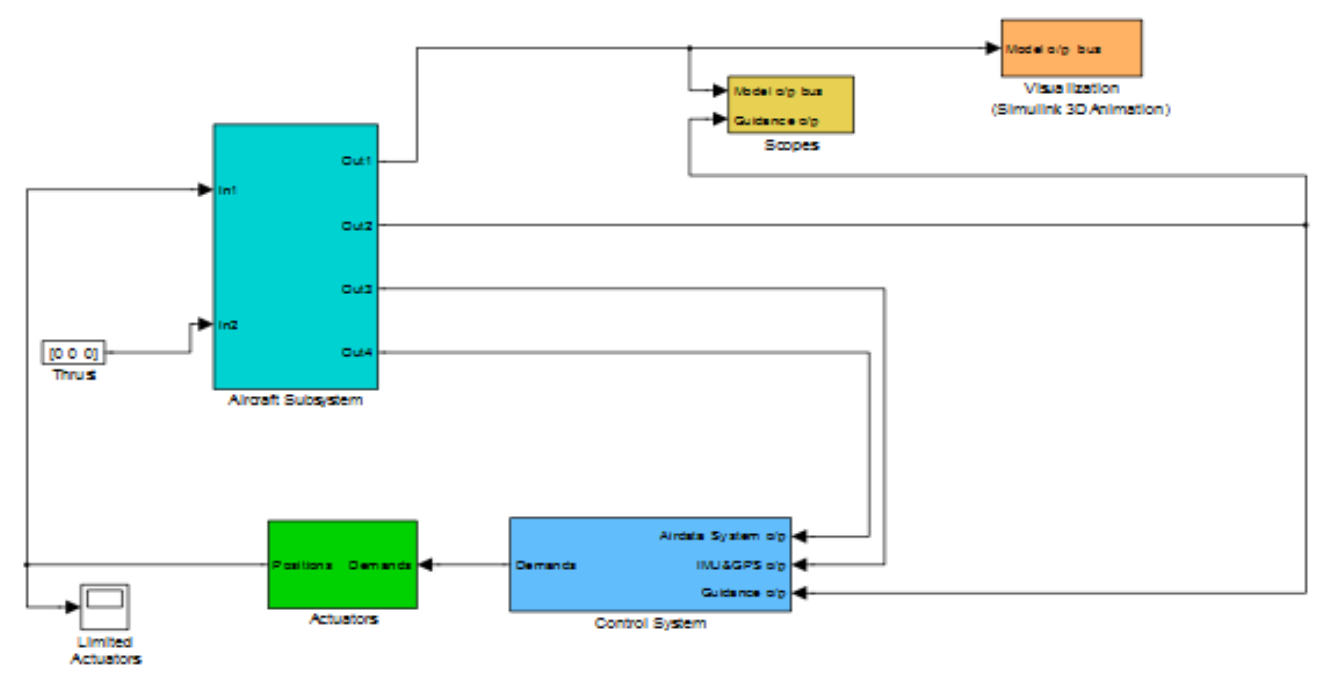

Table 1. Constant Block Properties

\begin{tabular}{|c|c|c|c|c|c|c|c|}
\hline Name & Value & Sampling Mode & Out Min & Out Max & Out Data Type Str & Sample Time & Frame Period \\
\hline Thrust & {$\left[\begin{array}{lll}0 & 0 & 0\end{array}\right]$} & Sample based & [] & [] & Inherit: Inherit from 'Constant value' & $\inf$ & $\inf$ \\
\hline
\end{tabular}

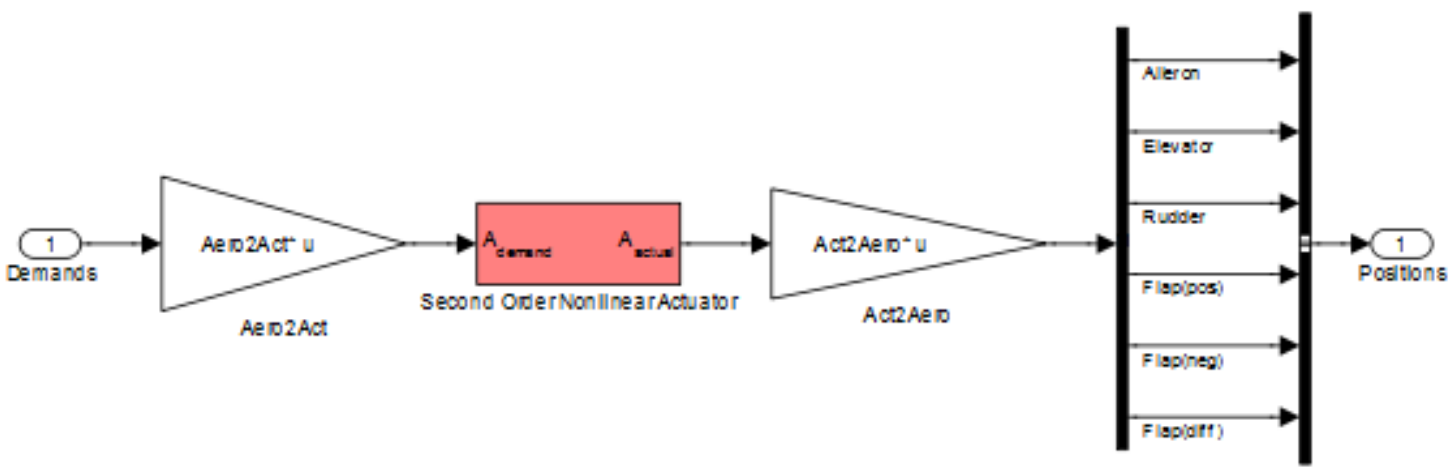

Table 2. BusCreator Block Properties

\begin{tabular}{|l|l|l|l|l|}
\hline Name & Inputs & Display Option & Bus Object & Non Virtual Bus \\
\hline Bus Creator1 & 6 & bar & BusObject & off \\
\hline
\end{tabular}

Table 3. Demux Block Properties

\begin{tabular}{|l|l|l|l|}
\hline Name & Outputs & Display Option & Bus Selection Mode \\
\hline Demux & 6 & none & off \\
\hline
\end{tabular}

Table 4. Gain Block Properties

\begin{tabular}{|c|c|c|c|c|c|c|c|c|}
\hline Name & Gain & Multiplication & Param Min & Param Max & $\begin{array}{l}\text { Param Data Type } \\
\text { Str }\end{array}$ & $\begin{array}{l}\text { Out } \\
\text { Min }\end{array}$ & Out Max & $\begin{array}{l}\text { Out Data Type } \\
\text { Str }\end{array}$ \\
\hline Name & \multirow{2}{*}{ Act2Aero } & \multirow{2}{*}{$\operatorname{Matrix}\left(\mathrm{K}^{*} \mathrm{u}\right)$} & \multirow{2}{*}{ [] } & \multirow{2}{*}{ [] } & \multirow{2}{*}{ Same as } & \multirow{2}{*}{ [] } & \multirow{2}{*}{ [] } & \multirow{2}{*}{$\begin{array}{l}\text { Inherit: Same as } \\
\text { input }\end{array}$} \\
\hline Act 2 Aero & & & & & & & & \\
\hline Aero2Act & Aero2Act & $\operatorname{Matrix}(\mathrm{K} * \mathrm{u})$ & [] & [] & $\begin{array}{l}\text { Inherit: Same as } \\
\text { input }\end{array}$ & [] & [] & $\begin{array}{l}\text { Inherit: Same as } \\
\text { input }\end{array}$ \\
\hline
\end{tabular}

Table 5. Inport Block Properties 


\begin{tabular}{|c|c|}
\hline Simulation Parameter & Value \\
\hline Solver & ode 45 \\
\hline RelTol & $1 \mathrm{e}-3$ \\
\hline Refine & 1 \\
\hline MaxOrder & 5 \\
\hline ZeroCross & on \\
\hline
\end{tabular}

Table 6. Outport Block Properties

\begin{tabular}{|c|c|c|c|c|c|c|c|c|c|c|c|}
\hline Name & Port & $\begin{array}{l}\text { Icon } \\
\text { Display }\end{array}$ & $\begin{array}{l}\text { Bus } \\
\text { Object }\end{array}$ & $\begin{array}{l}\text { Var } \\
\text { Size Sig }\end{array}$ & $\begin{array}{l}\text { Out } \\
\text { Min }\end{array}$ & $\begin{array}{l}\text { Out } \\
\text { Max }\end{array}$ & $\begin{array}{l}\text { Out } \\
\text { Data } \\
\text { Type Str }\end{array}$ & $\begin{array}{l}\text { Source } \\
\text { Of Initial } \\
\text { Output } \\
\text { Value }\end{array}$ & $\begin{array}{l}\text { Output } \\
\text { When } \\
\text { Disabled }\end{array}$ & $\begin{array}{l}\text { Initial } \\
\text { Outpu } \\
t\end{array}$ & $\begin{array}{l}\text { Used By } \\
\text { Blk }\end{array}$ \\
\hline Potions & 1 & $\begin{array}{l}\text { Port } \\
\text { number }\end{array}$ & $\begin{array}{l}\text { BusObje } \\
\text { ct }\end{array}$ & Inherit & [] & [] & $\begin{array}{l}\text { Inherit: } \\
\text { auto }\end{array}$ & Dialog & held & [] & $\begin{array}{l}\text { Abs2, } \\
\text { Product2, } \\
\text { Abs, } \\
\text { Product4, } \\
\text { Abs1, } \\
\text { Product6, } \\
\text { Product, } \\
\text { Product6, } \\
\text { Abs1, } \\
\text { Product4, } \\
\text { Abs, } \\
\text { Product2, } \\
\text { Abs2, } \\
\text { Product, } \\
\text { Product, } \\
\text { Abs2, } \\
\text { Product1, } \\
\text { Product2, } \\
\text { Product3, } \\
\text { Limited } \\
\text { Actuators }\end{array}$ \\
\hline
\end{tabular}

Table 7. Second Order Nonlinear Actuator Block Properties

\begin{tabular}{|l|l|l|l|l|l|l|}
\hline Name & Wn fin & Z fin & Fin $\max$ & Fin min & Fin maxrate & Fin act 0 \\
\hline Second Order Nonlinear Actuator & wn_act & z_act & max_lim & min_lim & inf & 0 \\
\hline
\end{tabular}

Table 8. BusCreator Block Properties

\begin{tabular}{|l|l|l|l|l|}
\hline Name & Inputs & Display Option & Bus Object & Non Virtual Bus \\
\hline Model o/p bus & 11 & bar & BusObject & off \\
\hline
\end{tabular}

Table 9. Inport Block Properties

\begin{tabular}{|l|l|l|l|l|}
\hline Name & Port & Defined In Blk & Out Min & Out Max \\
\hline In1 & 1 & Demux $, \underline{\text { Demux }}, \underline{\text { Demux }}, \underline{\text { Demux }, \underline{\text { Demux }}, \underline{\text { Demux }}}$ & {[]} & {[]} \\
\hline In2 & 2 & Thrust & {[]} & {[]} \\
\hline
\end{tabular}

\begin{tabular}{|c|c|c|c|c|c|c|c|c|c|c|c|}
\hline Name & Port & $\begin{array}{l}\text { Icon } \\
\text { Display }\end{array}$ & Bus Object & $\begin{array}{l}\text { Var Size } \\
\text { Sig }\end{array}$ & $\begin{array}{l}\text { Out } \\
\text { Min }\end{array}$ & $\begin{array}{l}\text { Out } \\
\text { Max }\end{array}$ & $\begin{array}{l}\text { Out Data } \\
\text { Type Str }\end{array}$ & $\begin{array}{l}\text { Source Of } \\
\text { Initial } \\
\text { Output } \\
\text { Value }\end{array}$ & $\begin{array}{l}\text { Output } \\
\text { When } \\
\text { Disable } \\
\text { d }\end{array}$ & $\begin{array}{l}\text { Initi } \\
\text { al } \\
\text { Outp } \\
\text { ut }\end{array}$ & Used By Blk \\
\hline Out1 & 1 & $\begin{array}{l}\text { Port } \\
\text { number }\end{array}$ & BusObject & Inherit & [] & [] & $\begin{array}{l}\text { Inherit: } \\
\text { auto }\end{array}$ & Dialog & held & [] & $\begin{array}{l}\text { Unit Conversion, } \\
\frac{\text { Product1 }, \quad \text { Demux, }}{\text { Demux, }} \\
\text { Conversion, } \\
\text { Attitudes } \\
\text { Accelerations }\end{array}$ \\
\hline
\end{tabular}




\begin{tabular}{|c|c|c|c|c|c|c|c|c|c|c|c|}
\hline & & & & & & & & & & & 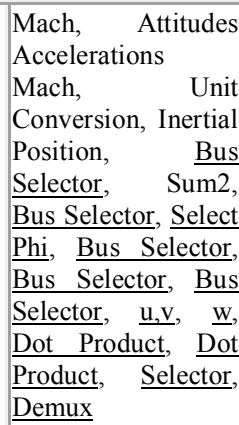 \\
\hline Out2 & 2 & $\begin{array}{l}\text { Port } \\
\text { number }\end{array}$ & BusObject & Inherit & [] & [] & $\begin{array}{l}\text { Inherit: } \\
\text { auto }\end{array}$ & Dialog & held & [] & $\begin{array}{l}\text { Demux2, Demux, } \\
{[1,4],[2,5], \underline{[3,6]}}\end{array}$ \\
\hline Out3 & 3 & $\begin{array}{l}\text { Port } \\
\text { number }\end{array}$ & BusObject & Inherit & [] & [] & $\begin{array}{l}\text { Inherit: } \\
\text { auto }\end{array}$ & Dialog & held & [] & Sum2, Sum1 \\
\hline Out4 & 4 & $\begin{array}{l}\text { Port } \\
\text { number }\end{array}$ & BusObject & Inherit & [] & [] & $\begin{array}{l}\text { Inherit: } \\
\text { auto }\end{array}$ & Dialog & held & [] & $\begin{array}{l}\text { Unit Conversion, } \\
\text { Product1 }\end{array}$ \\
\hline
\end{tabular}

Table 10. Outport Block Properties

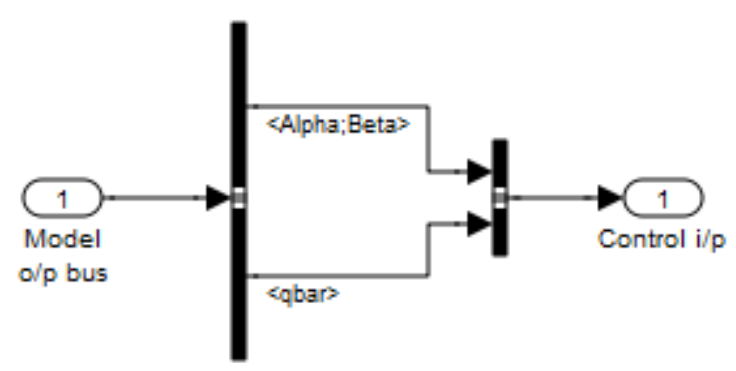

Table 11. BusCreator Block Properties

\begin{tabular}{|l|l|l|l|l|}
\hline Name & Inputs & Display Option & Bus Object & Non Virtual Bus \\
\hline Bus Creator1 & 2 & bar & BusObject & off \\
\hline
\end{tabular}

Table 12. BusSelector Block Properties

\begin{tabular}{|c|c|c|c|}
\hline Name & Output Signals & Output As Bus & Input Signals \\
\hline Bus Selector & Alpha;Beta,qbar & off & $\begin{array}{l}\text { pdot;qdot;rdot } \\
\text { pqr } \\
\text { phi;theta;psi } \\
\text { DCM } \\
\text { Accels } \\
\text { Vb } \\
\text { Ve } \\
\text { Xe } \\
\{\text { Alpha;Beta , Alpha Beta }\} \\
\text { Mach } \\
\text { qbar }\end{array}$ \\
\hline
\end{tabular}

Table 13. Inport Block Properties

\begin{tabular}{|l|l|l|l|l|l|}
\hline Name & Port & Defined In Blk & Out Min & Out Max \\
\hline $\begin{array}{l}\text { Model } \\
\text { bus }\end{array}$ & $\mathrm{o} / \mathrm{p}$ & 1 & $\begin{array}{l}\text { Reshape, p,q,r, phi theta psi, Reshape (9) to [3x3] column-major, Force ---> Acc, } \\
\begin{array}{l}\text { Unit Conversion, Unit Conversion, xe,ye,ze, Incidence, Sideslip, Product1, } \\
1 / 2 \mathrm{rhoV}^{\wedge} 2\end{array}\end{array}$ & {$\left[\begin{array}{l}{[]} \\
\hline\end{array}\right.$}
\end{tabular}


Table 14. Outport Block Properties

\begin{tabular}{|c|c|c|c|c|c|c|c|c|c|c|c|}
\hline Name & Port & $\begin{array}{l}\text { Icon } \\
\text { Display }\end{array}$ & Bus Object & $\begin{array}{l}\text { Var Size } \\
\text { Sig }\end{array}$ & $\begin{array}{l}\text { Out } \\
\text { Min }\end{array}$ & $\begin{array}{l}\text { Out } \\
\text { Max }\end{array}$ & $\begin{array}{l}\text { Out Data } \\
\text { Type Str }\end{array}$ & $\begin{array}{l}\text { Source Of } \\
\text { Initial } \\
\text { Output } \\
\text { Value }\end{array}$ & $\begin{array}{l}\text { Output } \\
\text { When } \\
\text { Disabled }\end{array}$ & $\begin{array}{l}\text { Initial } \\
\text { Output }\end{array}$ & Used By Blk \\
\hline
\end{tabular}

System - landing/Aircraft Subsystem/Guidance System

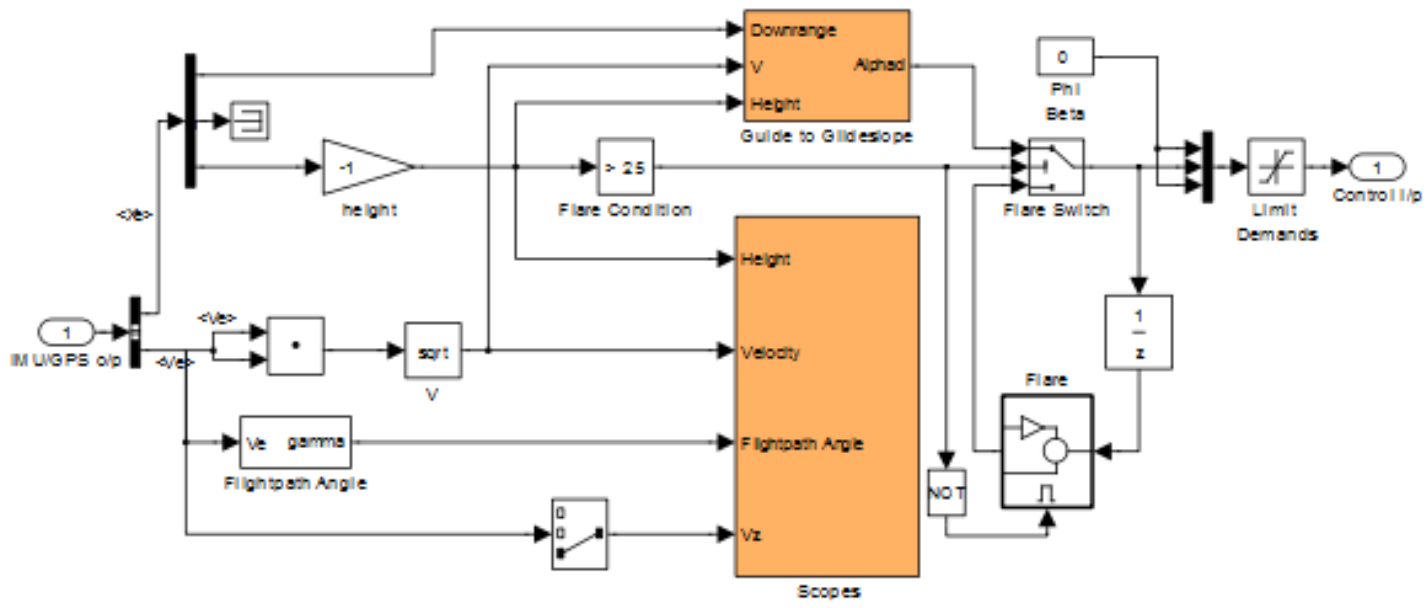

Table 15. BusSelector Block Properties

\begin{tabular}{|l|l|l|l|}
\hline Name & Output Signals & Output As Bus & Input Signals \\
\hline & & & pqr \\
Bus Selector & & phi; theta;psi \\
& & & DCM \\
& Xe,Ve & off & Accels \\
& & Vb \\
& & Ve \\
& & Xe \\
\hline
\end{tabular}

Table 16. Compare To Constant Block Properties

\begin{tabular}{|l|l|l|l|l|}
\hline Name & Relop & Const & Logic Out Data Type Mode & Zero Cross \\
\hline Flare Condition & $>$ & 25.0 & boolean & off \\
\hline
\end{tabular}

Table 17. Constant Block Properties

\begin{tabular}{|l|l|l|l|l|l|l|l|l|}
\hline Name & Value & Sampling Mode & Out Min & Out Max & Out Data Type Str & Sample Time & Frame Period \\
\hline Phi Beta & 0 & Sample based & {[]} & {[]} & Inherit: Inherit from 'Constant value' & inf & inf \\
\hline
\end{tabular}

Table 18. Demux Block Properties

\begin{tabular}{|l|l|l|l|}
\hline Name & Outputs & Display Option & Bus Selection Mode \\
\hline Demux & 3 & none & off \\
\hline
\end{tabular}

Table 19. DotProduct Block Properties

\begin{tabular}{|l|l|l|l|l|}
\hline Name & Input Same DT & Out Min & Out Max & Out Data Type Str \\
\hline Dot Product & off & {[]} & {[]} & Inherit: Inherit via internal rule \\
\hline
\end{tabular}

Table 20. Gain Block Properties

\begin{tabular}{|l|l|l|l|l|l|l|l|l|l|}
\hline Name & Gain & Multiplication & $\begin{array}{l}\text { Param } \\
\text { Min }\end{array}$ & $\begin{array}{l}\text { Param } \\
\text { Max }\end{array}$ & $\begin{array}{l}\text { Param Data Type } \\
\text { Str }\end{array}$ & Out Min & Out Max & Out Data Type Str \\
\hline height & -1 & Element-wise $(\mathrm{K} . * \mathrm{u})$ & {[]} & {[]} & Inherit: Same as input [] & {[]} & $\begin{array}{l}\text { Inherit: Same as } \\
\text { input }\end{array}$ \\
\hline
\end{tabular}


Table 21. Inport Block Properties

\begin{tabular}{|l|l|l|l|l|}
\hline Name & Port & Defined In Blk & Out Min & Out Max \\
\hline IMU/GPS o/p & 1 & $\begin{array}{l}\text { p,q,r }, \text { phi theta psi, Reshape (9) to [3x3] column-major, Force ---> Acc, Unit } \\
\text { Conversion, Unit Conversion, xe,ye,ze }\end{array}$ & {[]} & {[]}
\end{tabular}

Table 22. Logic Block Properties

\begin{tabular}{|l|l|l|l|l|l|}
\hline Name & Operator & Inputs & Icon Shape & $\begin{array}{l}\text { All Ports Same } \\
\text { DT }\end{array}$ & Out Data Type Str \\
\hline Logical Operator & NOT & 2 & rectangular & on & $\begin{array}{l}\text { Inherit: Logical (see Configuration } \\
\text { Parameters: Optimization) }\end{array}$ \\
\hline
\end{tabular}

Table 23. Math Block Properties

\begin{tabular}{|l|l|l|l|l|l|l|l|l|l|}
\hline Name & Operator & $\begin{array}{l}\text { Output Signal } \\
\text { Type }\end{array}$ & $\begin{array}{l}\text { Out } \\
\text { Min }\end{array}$ & $\begin{array}{l}\text { Out Data Type } \\
\text { Mtr }\end{array}$ & $\begin{array}{l}\text { Intermediate Results Data } \\
\text { Type Str }\end{array}$ & Algorithm Type & Iterations \\
\hline V & sqrt & auto & {[]} & {[]} & $\begin{array}{l}\text { Inherit: Same as } \\
\text { first input }\end{array}$ & $\begin{array}{l}\text { Inherit: Inherit via internal } \\
\text { rule }\end{array}$ & Newton-Raphson & 3 \\
\hline
\end{tabular}

Table 24. Mux Block Properties

\begin{tabular}{|l|l|l|}
\hline Name & Inputs & Display Option \\
\hline Mux & 3 & bar \\
\hline
\end{tabular}

Table 25. Outport Block Properties

\begin{tabular}{|c|c|c|c|c|c|c|c|c|c|c|c|}
\hline Name & Port & $\begin{array}{l}\text { Icon } \\
\text { Display }\end{array}$ & Bus Object & $\begin{array}{ll}\text { Var } & \text { Size } \\
\text { Sig } & \end{array}$ & $\begin{array}{l}\text { Out } \\
\text { Min }\end{array}$ & $\begin{array}{l}\text { Out } \\
\text { Max }\end{array}$ & $\begin{array}{l}\text { Out Data } \\
\text { Type Str }\end{array}$ & $\begin{array}{l}\text { Source Of } \\
\text { Initial } \\
\text { Output } \\
\text { Value }\end{array}$ & $\begin{array}{l}\text { Output } \\
\text { When } \\
\text { Disabled }\end{array}$ & $\begin{array}{l}\text { Initial } \\
\text { Output }\end{array}$ & Used By Blk \\
\hline Control i/p & 1 & $\begin{array}{l}\text { Port } \\
\text { number }\end{array}$ & BusObject & Inherit & [] & [] & $\begin{array}{l}\text { Inherit: } \\
\text { auto }\end{array}$ & Dialog & held & [] & $\begin{array}{l}\text { Demux2, } \\
\text { Demux, } \frac{[1,4],}{[2,5],[3,6]}\end{array}$ \\
\hline
\end{tabular}

Table 26. Saturate Block Properties

\begin{tabular}{|l|l|l|l|l|l|l|l|l|}
\hline Name & Upper Limit & Lower Limit & Linearize As Gain & Zero Cross & Out Min & Out Max & Out Data Type Str \\
\hline Limit Demands & {$\left[\begin{array}{llll}20 & 25 & 5\end{array}\right]$} & {$\left[\begin{array}{llll}-20 & -10 & -5\end{array}\right]$} & on & on & {[]} & {[]} & Inherit: Same as input \\
\hline
\end{tabular}

Table 27. Selector Block Properties

\begin{tabular}{|l|l|l|l|l|l|l|l|l|}
\hline Name & $\begin{array}{l}\text { Number Of Index } \\
\text { Dimensions }\end{array}$ & $\begin{array}{l}\text { Index Option } \\
\text { Mode }\end{array}$ & $\begin{array}{l}\text { Index } \\
\text { Array } \\
\text { Array }\end{array}$ & $\begin{array}{l}\text { Output Size } \\
\text { Array }\end{array}$ & $\begin{array}{l}\text { Input Port } \\
\text { Width }\end{array}$ & $\begin{array}{l}\text { Index } \\
\text { Options }\end{array}$ & $\begin{array}{l}\text { Ondices } \\
\text { Sizes }\end{array}$ \\
\hline Selector & 1 & $\begin{array}{l}\text { One- } \\
\text { based }\end{array}$ & $\begin{array}{l}\text { Index vector } \\
\text { (dialog) }\end{array}$ & {$[3]$} & 1 & 3 & $\begin{array}{l}\text { Index vector } \\
\text { (dialog) }\end{array}$ & {$[3]$} \\
\hline
\end{tabular}

Table 28. Switch Block Properties

\begin{tabular}{|l|l|l|l|l|l|l|l|l|}
\hline Name & Criteria & Threshold & $\begin{array}{l}\text { Input } \\
\text { Same DT }\end{array}$ & Out Min & Out Max & Out Data Type Str & Zero Cross & $\begin{array}{l}\text { Allow Diff Input } \\
\text { Sizes }\end{array}$ \\
\hline Flare Switch & $\mathrm{u} 2>=$ Threshold & 0.5 & on & {[]} & {[]} & $\begin{array}{l}\text { Inherit: Inherit via } \\
\text { internal rule }\end{array}$ & on \\
\hline
\end{tabular}

Table 29. Terminator Block Properties

\begin{tabular}{|l|}
\hline Name \\
\hline Terminator
\end{tabular}

Table 30. UnitDelay Block Properties

\begin{tabular}{|l|l|l|}
\hline Name & X0 & Sample Time \\
\hline Unit Delay & 0 & 0.1 \\
\hline
\end{tabular}

System - landing/Aircraft Subsystem/Guidance System/Flare 


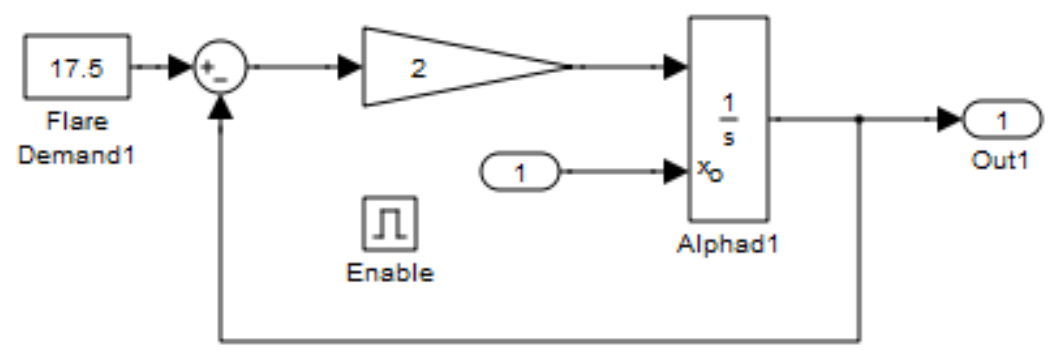

Table 31. Constant Block Properties

\begin{tabular}{|l|l|l|l|l|l|l|l|l|}
\hline Name & Value & Sampling Mode & Out Min & Out Max & Out Data Type Str & Sample Time & Frame Period \\
\hline Flare Demand1 & 17.5 & Sample based & {[]} & {[]} & $\begin{array}{l}\text { Inherit: Inherit from } \\
\text { 'Constant value' }\end{array}$ & inf \\
\hline
\end{tabular}

Table 32. EnablePort Block Properties

\begin{tabular}{|l|l|l|l|l|}
\hline Name & States When Enabling & Propagate Var Size & Show Output Port & Zero Cross \\
\hline Enable & reset & Only when enabling & off & on \\
\hline
\end{tabular}

Table 33. Gain Block Properties

\begin{tabular}{|l|l|l|l|l|l|l|l|l|l|}
\hline Name & Gain & Multiplication & $\begin{array}{l}\text { Param } \\
\text { Min }\end{array}$ & $\begin{array}{l}\text { Param } \\
\text { Max }\end{array}$ & $\begin{array}{l}\text { Param Data Type } \\
\text { Str }\end{array}$ & Out Min & Out Max & Out Data Type Str \\
\hline Gain1 & 2 & Element-wise(K.*u) & {[]} & {[]} & Inherit: Same as input & {[]} & {[]} & $\begin{array}{l}\text { Inherit: } \\
\text { input }\end{array}$ \\
\hline
\end{tabular}

Table 34. Inport Block Properties

\begin{tabular}{|l|l|l|l|l|}
\hline Name & Port & Defined In BIk & Out Min & Out Max \\
\hline In1 & 1 & Unit Delay & {[]} & {[]} \\
\hline
\end{tabular}

Table 35. Integrator Block Properties

\begin{tabular}{|l|l|l|l|l|}
\hline Name & External Reset & Initial Condition Source & Zero Cross & Continuous State Attributes \\
\hline Alphad1 & none & external & on & $"$ \\
\hline
\end{tabular}

Table 36. Outport Block Properties

\begin{tabular}{|c|c|c|c|c|c|c|c|c|c|c|c|}
\hline Name & Port & $\begin{array}{l}\text { Icon } \\
\text { Display }\end{array}$ & Bus Object & $\begin{array}{ll}\text { Var } & \text { Size } \\
\text { Sig } & \end{array}$ & $\begin{array}{l}\text { Out } \\
\text { Min }\end{array}$ & $\begin{array}{l}\text { Out } \\
\text { Max }\end{array}$ & $\begin{array}{l}\text { Out Data } \\
\text { Type Str }\end{array}$ & \begin{tabular}{|l|} 
Source \\
Initial \\
Output \\
Value
\end{tabular} & $\begin{array}{l}\text { Output When } \\
\text { Disabled }\end{array}$ & $\begin{array}{l}\text { Initial } \\
\text { Output }\end{array}$ & $\begin{array}{l}\text { Used By } \\
\text { Blk }\end{array}$ \\
\hline Out1 & 1 & Port number & BusObject & Inherit & [] & [] & $\begin{array}{l}\text { Inherit: } \\
\text { auto }\end{array}$ & Dialog & held & {$[0]$} & $\begin{array}{l}\frac{\text { Flare }}{\text { Switch }} \\
\text { Sum2 }\end{array}$ \\
\hline
\end{tabular}

Table 37. Sum Block Properties

\begin{tabular}{|c|c|c|c|c|c|c|c|c|c|}
\hline Name & Icon Shape & Inputs & Collapse Mode & Collapse Dim & $\begin{array}{l}\text { Input Same } \\
\text { DT }\end{array}$ & $\begin{array}{l}\text { Accum Data Type } \\
\text { Str }\end{array}$ & $\begin{array}{l}\text { Out } \\
\text { Min }\end{array}$ & $\begin{array}{l}\text { Out } \\
\text { Max }\end{array}$ & $\begin{array}{l}\text { Out Data Type } \\
\text { Str }\end{array}$ \\
\hline Sum2 & round & |+- & All dimensions & 1 & on & $\begin{array}{l}\text { Inherit: Inherit via } \\
\text { internal rule }\end{array}$ & [] & [] & $\begin{array}{l}\text { Inherit: Same as } \\
\text { first input }\end{array}$ \\
\hline
\end{tabular}

System - landing/Aircraft Subsystem/Guidance System/Flightpath Angle

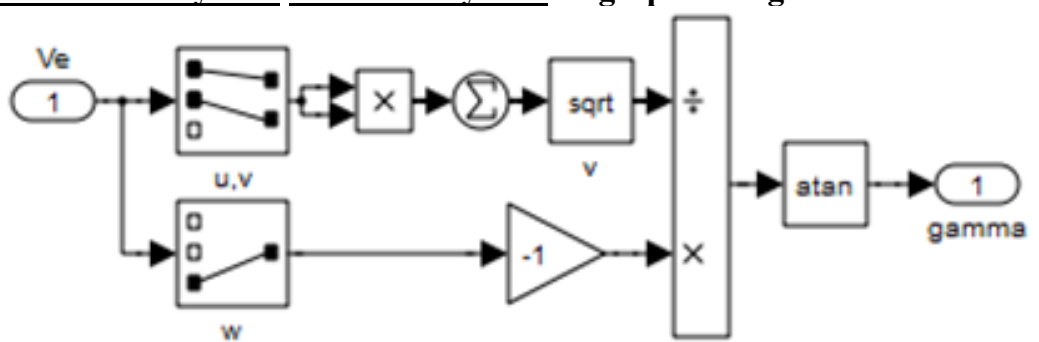

Table 38. Gain Block Properties 
Take Off And Landing Stage Of Aircraft with Matlab Simulation

\begin{tabular}{|c|c|c|c|c|c|c|c|c|c|}
\hline Name & Gain & Multiplication & Param Min & Param Max & $\begin{array}{l}\text { Param Data } \\
\text { Type Str }\end{array}$ & Out Min & Out Max & $\begin{array}{l}\text { Out } \\
\text { Type Str }\end{array}$ & Data \\
\hline rad-->deg4 & -1 & Element-wise $\left(\mathrm{K} .{ }^{*} \mathrm{u}\right)$ & [] & [] & $\begin{array}{l}\text { Inherit: Same } \\
\text { as input }\end{array}$ & [] & [] & $\begin{array}{l}\text { Inherit: } \\
\text { as input }\end{array}$ & Same \\
\hline
\end{tabular}

Table 39. Inport Block Properties

\begin{tabular}{|l|l|l|l|l|}
\hline Name & Port & Defined In Blk & Out Min & Out Max \\
\hline Ve & 1 & Unit Conversion & {[]} & {[]} \\
\hline
\end{tabular}

Table 40. Math Block Properties

\begin{tabular}{|l|l|l|l|l|l|l|l|l|l|}
\hline Name & Operator & $\begin{array}{l}\text { Output Signal } \\
\text { Type }\end{array}$ & $\begin{array}{l}\text { Out } \\
\text { Min }\end{array}$ & $\begin{array}{l}\text { Out } \\
\text { Max }\end{array}$ & $\begin{array}{l}\text { Out Data Type } \\
\text { Str }\end{array}$ & $\begin{array}{l}\text { Intermediate Results Data } \\
\text { Type Str }\end{array}$ & Algorithm Type & Iterations \\
\hline V & sqrt & auto & {[]} & {[]} & $\begin{array}{l}\text { Inherit: Same as } \\
\text { first input }\end{array}$ & $\begin{array}{l}\text { Inherit: Inherit via internal } \\
\text { rule }\end{array}$ & Newton-Raphson & 3 \\
\hline
\end{tabular}

Table 41. Outport Block Properties

\begin{tabular}{|l|l|l|l|l|l|l|l|l|l|l|l|l|l|l|l}
\hline Name & Port & $\begin{array}{l}\text { Icon } \\
\text { Display }\end{array}$ & Bus Object & $\begin{array}{l}\text { Var Size } \\
\text { Sig }\end{array}$ & $\begin{array}{l}\text { Out } \\
\text { Min }\end{array}$ & $\begin{array}{l}\text { Out } \\
\text { Max }\end{array}$ & $\begin{array}{l}\text { Out Data Of } \\
\text { Type Str }\end{array}$ & $\begin{array}{l}\text { Initial } \\
\text { Output } \\
\text { Value }\end{array}$ & $\begin{array}{l}\text { Output } \\
\text { When } \\
\text { Disabled }\end{array}$ & $\begin{array}{l}\text { Initial } \\
\text { Output }\end{array}$ & Used By Blk \\
\hline gamma & 1 & $\begin{array}{l}\text { Port } \\
\text { number }\end{array}$ & BusObject & Inherit & {[]} & {[]} & $\begin{array}{l}\text { Inherit: } \\
\text { auto }\end{array}$ & Dialog & held & {[]} & Unit Conversion \\
\hline
\end{tabular}

Table 42. Product Block Properties

\begin{tabular}{|l|l|l|l|l|l|l|l|l|}
\hline Name & Inputs & Multiplication & Collapse Mode & Collapse Dim & $\begin{array}{l}\text { Input } \\
\text { Same } \\
\text { DT }\end{array}$ & $\begin{array}{l}\text { Out } \\
\text { Min }\end{array}$ & Out Max & Out Data Type Str \\
\hline product & 2 & Element-wise(.*) & All dimensions & 1 & on & {[]} & {[]} & $\begin{array}{l}\text { Inherit: Same as first } \\
\text { input }\end{array}$ \\
\hline product1 & $/ *$ & Element-wise(.*) & All dimensions & 1 & on & {[]} & {[]} & $\begin{array}{l}\text { Inherit: Same as first } \\
\text { input }\end{array}$ \\
\hline
\end{tabular}

Table 43. Selector Block Properties

\begin{tabular}{|c|c|c|c|c|c|c|c|c|c|}
\hline Name & $\begin{array}{l}\text { Number } \\
\text { Dimensions }\end{array}$ & Of $\begin{array}{l}\text { Index } \\
\text { Mode }\end{array}$ & $\begin{array}{l}\text { Index Option } \\
\text { Array }\end{array}$ & $\begin{array}{l}\text { Index } \\
\text { Param } \\
\text { Array }\end{array}$ & $\begin{array}{l}\text { Output } \\
\text { Size } \\
\text { Array }\end{array}$ & \begin{tabular}{|l} 
Input \\
Port \\
Width
\end{tabular} & Index Options & Indices & $\begin{array}{l}\text { Output } \\
\text { Sizes }\end{array}$ \\
\hline $\mathrm{u}, \mathrm{v}$ & 1 & $\begin{array}{l}\text { One- } \\
\text { based }\end{array}$ & $\begin{array}{l}\text { Index vector } \\
(\text { dialog) }\end{array}$ & {$\left[\begin{array}{ll}1 & 2\end{array}\right]$} & 1 & 3 & $\begin{array}{ll}\text { Index } & \text { vector } \\
(\text { dialog) } & \end{array}$ & {$\left[\begin{array}{ll}1 & 2\end{array}\right]$} & 1 \\
\hline $\mathrm{w}$ & 1 & $\begin{array}{l}\text { One- } \\
\text { based }\end{array}$ & $\begin{array}{l}\text { Index vector } \\
\text { (dialog) }\end{array}$ & 3 & 1 & 3 & $\begin{array}{l}\text { Index vector } \\
\text { (dialog) }\end{array}$ & 3 & 1 \\
\hline
\end{tabular}

Table 44. Sum Block Properties

\begin{tabular}{|c|c|c|c|c|c|c|c|c|c|}
\hline Name & Icon Shape & Inputs & Collapse Mode & Collapse Dim & $\begin{array}{l}\text { Input } \\
\text { Same } \\
\text { DT }\end{array}$ & $\begin{array}{l}\text { Accum } \\
\text { Type Str }\end{array}$ & $\begin{array}{l}\text { Out } \\
\text { Min }\end{array}$ & $\begin{array}{l}\text { Out } \\
\text { Max }\end{array}$ & $\begin{array}{lll}\text { Out Data } & \text { Type } \\
\text { Str } & & \end{array}$ \\
\hline Sum2 & round & + & All dimensions & 1 & on & $\begin{array}{l}\text { Inherit: Inherit } \\
\text { via internal rule }\end{array}$ & [] & [] & $\begin{array}{l}\text { Inherit: Same as } \\
\text { first input }\end{array}$ \\
\hline
\end{tabular}

Table 45. Trigonometry Block Properties

\begin{tabular}{|l|l|l|l|l|}
\hline Name & Operator & Approximation Method & Number Of Iterations & Output Signal Type \\
\hline tangent & atan & None & 11 & auto \\
\hline
\end{tabular}

System - landing/Aircraft Subsystem/Guidance System/Guide to Glideslope

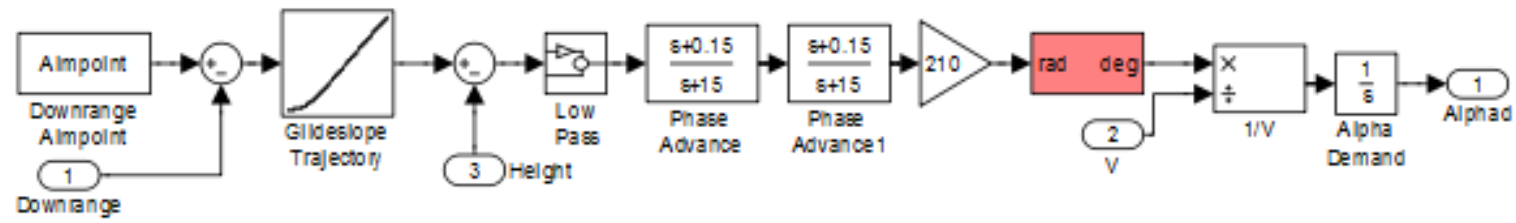


Table 46. Angle Conversion Block Properties

\begin{tabular}{|l|l|l|}
\hline Name & IU & OU \\
\hline Angle Conversion & rad & deg \\
\hline
\end{tabular}

Table 47. Constant Block Properties

\begin{tabular}{|c|c|c|c|c|c|c|c|}
\hline Name & Value & Sampling Mode & Out Min & Out Max & Out Data Type Str & Sample Time & Frame Period \\
\hline Downrange Aimpoint & Aimpoint & Sample based & [] & [] & $\begin{array}{l}\text { Inherit: Inherit from } \\
\text { 'Constant value' }\end{array}$ & inf & inf \\
\hline
\end{tabular}

Table 48. Gain Block Properties

\begin{tabular}{|l|l|l|l|l|l|l|l|l|}
\hline Name & Gain & Multiplication & Param Min & Param Max & $\begin{array}{l}\text { Param Data Type } \\
\text { Str }\end{array}$ & Out Min & Out Max & $\begin{array}{l}\text { Out } \\
\text { Type Str }\end{array}$ \\
\hline Gain4 & 210 & Element-wise(K.*u) & {[]} & {[]} & Inherit: Same as input & {[]} & {[]} & $\begin{array}{l}\text { Inherit: } \\
\text { as input }\end{array}$ \\
\hline
\end{tabular}

Table 49. Inport Block Properties

\begin{tabular}{|c|c|c|c|c|}
\hline Name & Port & Defined In BIk & Out Min & Out Max \\
\hline Downrange & 1 & Demux & [] & [] \\
\hline Height & 3 & height & {[]} & [] \\
\hline V & 2 & $\underline{\mathrm{V}}$ & [] & [] \\
\hline
\end{tabular}

Table 50. Integrator Block Properties

\begin{tabular}{|l|l|l|l|l|l|}
\hline Name & External Reset & Initial Condition Source & Initial Condition & Zero Cross & Continuous State Attributes \\
\hline Alpha Demand & none & internal & alpha0*180/pi & on & $"$ \\
\hline
\end{tabular}

Table 51. Lookup Block Properties

\begin{tabular}{|l|l|l|l|l|l|l|l|}
\hline Name & Input Values & Table & Look Up Meth & Out Min & Out Max & Out Data Type Str \\
\hline Glideslope Trajectory & Xglide & hglide & Interpolation-Extrapolation & {[]} & {[]} & Inherit: Same as input \\
\hline
\end{tabular}

Table 52. Outport Block Properties

\begin{tabular}{|c|c|c|c|c|c|c|c|c|c|c|c|}
\hline Name & Port & $\begin{array}{l}\text { Icon } \\
\text { Display }\end{array}$ & Bus Object & $\begin{array}{ll}\text { Var } & \text { Size } \\
\text { Sig } & \end{array}$ & $\begin{array}{l}\text { Out } \\
\text { Min }\end{array}$ & $\begin{array}{l}\text { Out } \\
\text { Max }\end{array}$ & $\begin{array}{l}\text { Out Data } \\
\text { Type Str }\end{array}$ & \begin{tabular}{|l|} 
Source \\
Initial \\
Output \\
Value
\end{tabular} & $\begin{array}{l}\text { Output When } \\
\text { Disabled }\end{array}$ & $\begin{array}{l}\text { Initial } \\
\text { Output }\end{array}$ & $\begin{array}{ll}\text { Used } & \text { By } \\
\text { Blk } & \end{array}$ \\
\hline Alphad & 1 & Port number & BusObject & Inherit & [] & [] & $\begin{array}{l}\text { Inherit: } \\
\text { auto }\end{array}$ & Dialog & held & [] & 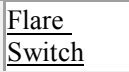 \\
\hline
\end{tabular}

Table 53. Product Block Properties

\begin{tabular}{|l|l|l|l|l|l|l|l|l|}
\hline Name & Inputs & Multiplication & Collapse Mode & Collapse Dim & $\begin{array}{l}\text { Input Same } \\
\text { DT }\end{array}$ & Out Min & Out Max & Out Data Type Str \\
\hline $1 / \mathrm{V}$ & $* /$ & Element-wise(.*) & All dimensions & 1 & on & {[]} & {[]} & $\begin{array}{l}\text { Inherit: Same as first } \\
\text { input }\end{array}$ \\
\hline
\end{tabular}

Table 54. Sum Block Properties

\begin{tabular}{|c|c|c|c|c|c|c|c|c|c|}
\hline Name & Icon Shape & Inputs & Collapse Mode & Collapse Dim & $\begin{array}{l}\text { Input Same } \\
\text { DT }\end{array}$ & \begin{tabular}{|l|} 
Accum Data \\
Type Str
\end{tabular} & $\begin{array}{l}\text { Out } \\
\text { Min }\end{array}$ & $\begin{array}{l}\text { Out } \\
\text { Max }\end{array}$ & $\begin{array}{l}\text { Out Data Type } \\
\text { Str }\end{array}$ \\
\hline Sum1 & round & |+- & All dimensions & 1 & on & $\begin{array}{l}\text { Inherit: Inherit } \\
\text { via internal rule }\end{array}$ & [] & [] & $\begin{array}{l}\text { Inherit: Same as } \\
\text { first input }\end{array}$ \\
\hline Sum2 & round & |+- & All dimensions & 1 & on & $\begin{array}{l}\text { Inherit: Inherit } \\
\text { via internal rule }\end{array}$ & [] & [] & $\begin{array}{l}\text { Inherit: Same as } \\
\text { first input }\end{array}$ \\
\hline
\end{tabular}

Table 55. TransferFcn Block Properties

\begin{tabular}{|l|l|l|l|}
\hline Name & Numerator & Denominator & Continuous State Attributes \\
\hline Phase Advance & {$\left[\begin{array}{ll}1 & 0.15\end{array}\right]$} & {$\left[\begin{array}{ll}1 & 15\end{array}\right]$} & $"$ \\
\hline Phase Advance1 & {$\left[\begin{array}{lll}1 & 0.15\end{array}\right]$} & {$\left[\begin{array}{ll}1 & 15\end{array}\right]$} & $"$ \\
\hline
\end{tabular}

System - landing/Aircraft Subsystem/Guidance System/Guide to Glideslope/Low Pass 


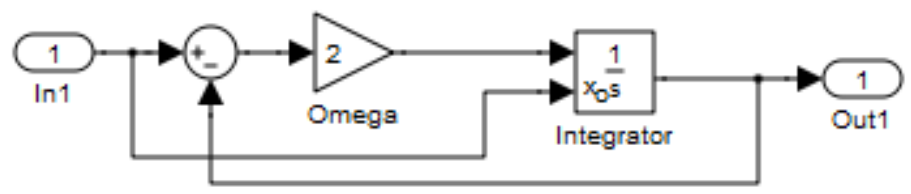

Table 56. Gain Block Properties

\begin{tabular}{|l|l|l|l|l|l|l|l|l|}
\hline Name & Gain & Multiplication & Param Min & Param Max & $\begin{array}{l}\text { Param Data Type } \\
\text { Str }\end{array}$ & Out Min & Out Max & Out Data Type Str \\
\hline Omega & 2 & Element-wise $(K . * \mathrm{u})$ & {[]} & {[]} & Inherit: Same as input & {[]} & {$\left[\begin{array}{llll}{[} & \text { Inherit: } & \text { Same } \\
\text { input }\end{array}\right.$} \\
\hline
\end{tabular}

Table 57. Inport Block Properties

\begin{tabular}{|l|l|l|l|l|}
\hline Name & Port & Defined In Blk & Out Min & Out Max \\
\hline In1 & 1 & $\underline{\text { Sum2 }}$ & {[]} & {[]} \\
\hline
\end{tabular}

Table 58. Integrator Block Properties

\begin{tabular}{|l|l|l|l|l|}
\hline Name & External Reset & Initial Condition Source & Zero Cross & Continuous State Attributes \\
\hline Integrator & none & external & on & $"$ \\
\hline
\end{tabular}

Table 59. Outport Block Properties

\begin{tabular}{|c|c|c|c|c|c|c|c|c|c|c|c|}
\hline Name & Port & $\begin{array}{l}\text { Icon } \\
\text { Display }\end{array}$ & Bus Object & $\begin{array}{l}\text { Var Size } \\
\text { Sig }\end{array}$ & $\begin{array}{l}\text { Out } \\
\text { Min }\end{array}$ & $\begin{array}{l}\text { Out } \\
\text { Max }\end{array}$ & $\begin{array}{l}\text { Out Data } \\
\text { Type Str }\end{array}$ & $\begin{array}{|ll|}\text { Source Of } \\
\text { Initial } \\
\text { Output } \\
\text { Value }\end{array}$ & $\begin{array}{l}\text { Output } \\
\text { When } \\
\text { Disabled }\end{array}$ & $\begin{array}{l}\text { Initial } \\
\text { Output }\end{array}$ & Used By Blk \\
\hline Out1 & 1 & $\begin{array}{l}\text { Port } \\
\text { number }\end{array}$ & BusObject & Inherit & [] & [] & $\begin{array}{l}\text { Inherit: } \\
\text { auto }\end{array}$ & Dialog & held & [] & $\begin{array}{l}\frac{\text { Phase }}{\text { Advance, }} \\
\underline{\text { Sum3 }}\end{array}$ \\
\hline
\end{tabular}

Table 60. Sum Block Properties

\begin{tabular}{|c|c|c|c|c|c|c|c|c|c|}
\hline Name & Icon Shape & Inputs & Collapse Mode & Collapse Dim & $\begin{array}{l}\text { Input Same } \\
\text { DT }\end{array}$ & $\begin{array}{l}\text { Accum Data Type } \\
\text { Str }\end{array}$ & $\begin{array}{l}\text { Out } \\
\text { Min }\end{array}$ & $\begin{array}{l}\text { Out } \\
\text { Max }\end{array}$ & $\begin{array}{l}\text { Out Data Type } \\
\text { Str }\end{array}$ \\
\hline Sum3 & round & H- & All dimensions & 1 & on & $\begin{array}{l}\text { Inherit: Inherit via } \\
\text { internal rule }\end{array}$ & [] & [] & $\begin{array}{l}\text { Inherit: Same as } \\
\text { first input }\end{array}$ \\
\hline
\end{tabular}

System - landing/Aircraft Subsystem/Guidance System/Scopes

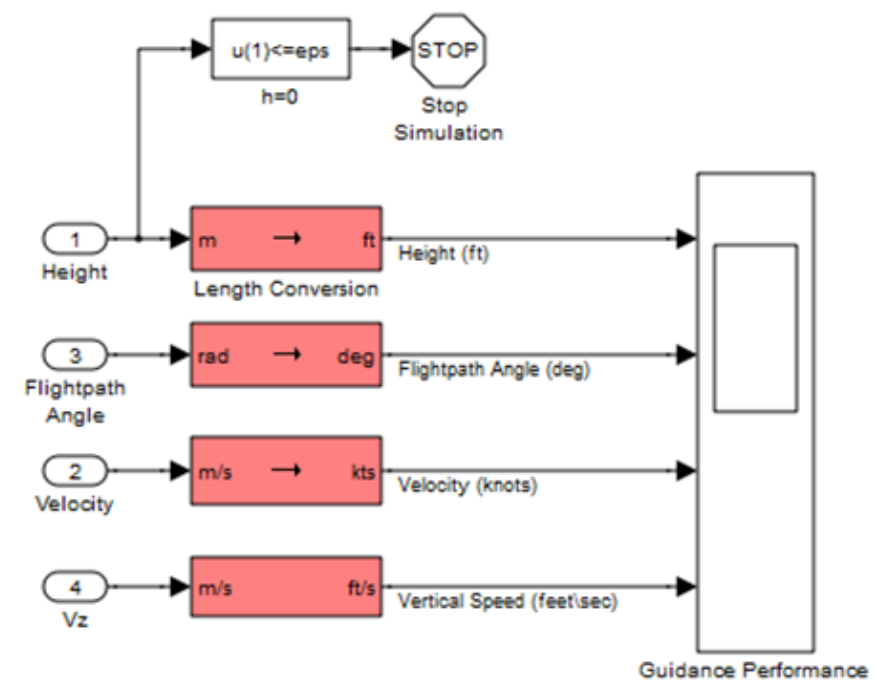

Table 61. Angle Conversion Block Properties

\begin{tabular}{|l|l|l|}
\hline Name & IU & OU \\
\hline Angle Conversion & rad & deg \\
\hline
\end{tabular}


Table 62. Fcn Block Properties

\begin{tabular}{|l|l|}
\hline Name & Expr \\
\hline $\mathrm{h}=0$ & $\mathrm{u}(1)<=$ eps \\
\hline
\end{tabular}

Table 63. Inport Block Properties

\begin{tabular}{|c|c|c|c|c|}
\hline Name & Port & Defined In Blk & Out Min & Out Max \\
\hline Flightpath Angle & 3 & tangent & [] & [] \\
\hline Height & 1 & height & [] & [] \\
\hline Velocity & 2 & $\underline{\mathrm{V}}$ & [] & [] \\
\hline $\mathrm{Vz}$ & 4 & Selector & [] & [] \\
\hline
\end{tabular}

Table 64. Length Conversion Block Properties

\begin{tabular}{|l|l|l|}
\hline Name & IU & OU \\
\hline Length Conversion & $\mathrm{m}$ & $\mathrm{ft}$ \\
\hline
\end{tabular}

Table 65. Stop Block Properties

Name

Stop Simulation

Table 66. Velocity Conversion Block Properties

\begin{tabular}{|l|l|l|}
\hline Name & IU & OU \\
\hline V2 & $\mathrm{m} / \mathrm{s}$ & $\mathrm{kts}$ \\
\hline Velocity Conversion & $\mathrm{m} / \mathrm{s}$ & $\mathrm{ft} / \mathrm{s}$ \\
\hline
\end{tabular}

System - landing/Aircraft Subsystem/IMU,GPS \& Radar Altimeter

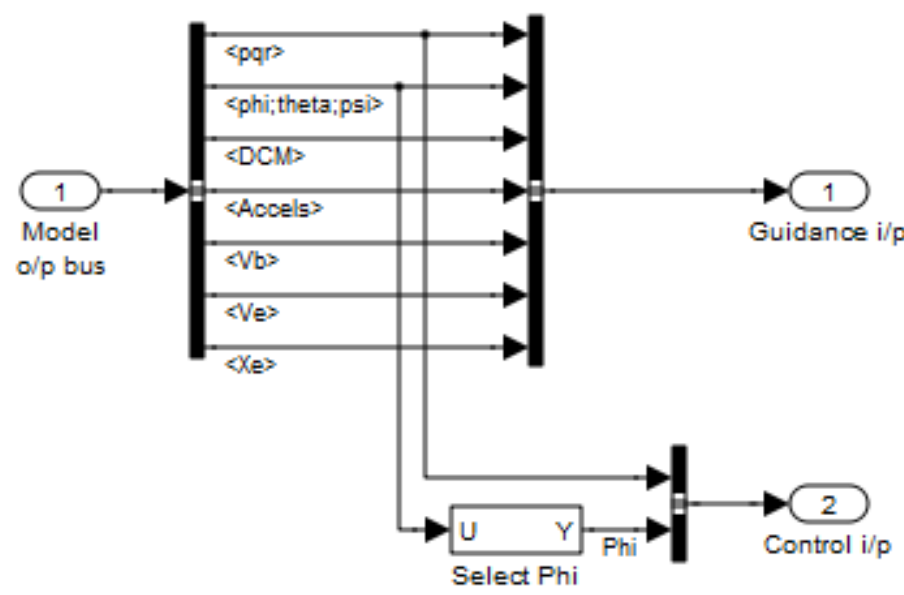

Table 67. BusCreator Block Properties

\begin{tabular}{|l|l|l|l|l|}
\hline Name & Inputs & Display Option & Bus Object & Non Virtual Bus \\
\hline Bus Creator & 7 & bar & BusObject & off \\
\hline Bus Creator1 & 2 & bar & BusObject & off \\
\hline
\end{tabular}


Table 68. BusSelector Block Properties

\begin{tabular}{|l|l|l|l|}
\hline Name & Output Signals & Output As Bus & Input Signals \\
\hline & & pdot;qdot;rdot \\
pqr \\
phi;theta;psi \\
DucM Selector
\end{tabular}

Table 69. Inport Block Properties

\begin{tabular}{|c|c|c|c|c|}
\hline Name & Port & Defined In Blk & $\begin{array}{l}\text { Out } \\
\text { Min }\end{array}$ & Out Max \\
\hline $\begin{array}{ll}\text { Model } & \mathrm{o} / \mathrm{p} \\
\text { bus }\end{array}$ & 1 & $\begin{array}{l}\text { Reshape, p,q,r, phi theta psi, Reshape (9) to [3x3] column-major, Force ---> Acc, Unit } \\
\text { Conversion, Unit Conversion, xe,ye,ze, Incidence, Sideslip, Product1, } 1 / 2 \mathrm{rhoV}^{\wedge} 2\end{array}$ & [] & [] \\
\hline
\end{tabular}

Table 70. Outport Block Properties

\begin{tabular}{|c|c|c|c|c|c|c|c|c|c|c|c|}
\hline Name & Port & $\begin{array}{l}\text { Icon } \\
\text { Display }\end{array}$ & Bus Object & $\begin{array}{l}\text { Var Size } \\
\text { Sig }\end{array}$ & $\begin{array}{l}\text { Out } \\
\text { Min }\end{array}$ & $\begin{array}{l}\text { Out } \\
\text { Max }\end{array}$ & $\begin{array}{l}\text { Out Data } \\
\text { Type Str }\end{array}$ & \begin{tabular}{|l|} 
Source Of \\
Initial \\
Output \\
Value
\end{tabular} & $\begin{array}{l}\text { Output } \\
\text { When } \\
\text { Disabled }\end{array}$ & $\begin{array}{l}\text { Initial } \\
\text { Output }\end{array}$ & $\begin{array}{l}\text { Used } \quad \text { By } \\
\text { Blk }\end{array}$ \\
\hline Guidance i/p & 1 & $\begin{array}{l}\text { Port } \\
\text { number }\end{array}$ & BusObject & Inherit & [] & [] & $\begin{array}{l}\text { Inherit: } \\
\text { auto }\end{array}$ & Dialog & held & [] & $\begin{array}{l}\frac{\text { Demux }}{\mathrm{w}}, \frac{\mathrm{u}, \mathrm{v}}{\mathrm{Dot}}, \\
\frac{\text { Product }}{\text { Dot }} \\
\underline{\text { Product }}, \\
\text { Selector }\end{array}$ \\
\hline
\end{tabular}

Table 71. Selector Block Properties

\begin{tabular}{|c|c|c|c|c|c|c|c|c|c|}
\hline Name & $\begin{array}{l}\text { Number Of } \\
\text { Dimensions }\end{array}$ & $\begin{array}{l}\text { Index } \\
\text { Mode }\end{array}$ & $\begin{array}{l}\text { Index Option } \\
\text { Array }\end{array}$ & $\begin{array}{l}\text { Index } \\
\text { Param } \\
\text { Array }\end{array}$ & $\begin{array}{l}\text { Output Size } \\
\text { Array }\end{array}$ & $\begin{array}{l}\text { Input Port } \\
\text { Width }\end{array}$ & Index Options & Indices & $\begin{array}{l}\text { Output } \\
\text { Sizes }\end{array}$ \\
\hline Select Phi & 1 & $\begin{array}{l}\text { One- } \\
\text { based }\end{array}$ & $\begin{array}{l}\text { Index vector } \\
(\text { dialog })\end{array}$ & {$[1]$} & 1 & 3 & $\begin{array}{l}\text { Index vector } \\
\text { (dialog) }\end{array}$ & [1] & 1 \\
\hline
\end{tabular}

System - landing/Control System

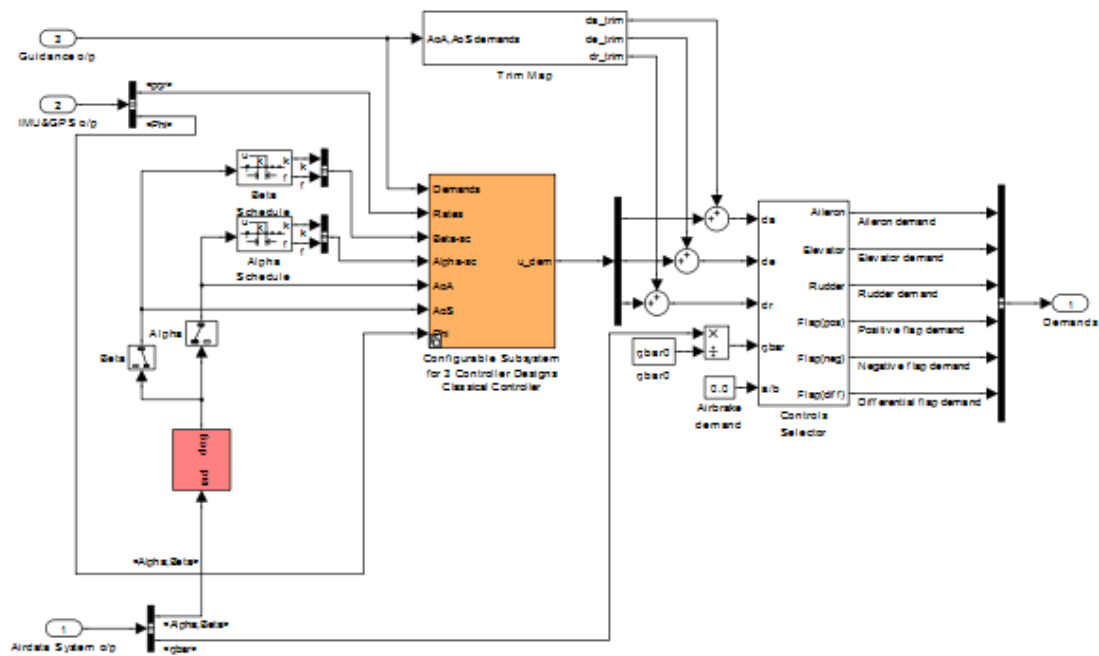


Table 72. Angle Conversion Block Properties

\begin{tabular}{|l|l|l|}
\hline Name & IU & OU \\
\hline Angle Conversion & rad & deg \\
\hline
\end{tabular}

Table 73. BusCreator Block Properties

\begin{tabular}{|l|l|l|l|l|}
\hline Name & Inputs & Display Option & Bus Object & Non Virtual Bus \\
\hline Bus Creator & 2 & bar & BusObject & off \\
\hline Bus Creator1 & 6 & bar & BusObject & off \\
\hline Bus Creator2 & 2 & bar & BusObject & off \\
\hline
\end{tabular}

Table 74. BusSelector Block Properties

\begin{tabular}{|l|l|l|l|}
\hline Name & Output Signals & Output As Bus & Input Signals \\
\hline Bus Selector & pqr,Phi & off & $\begin{array}{l}\text { pqr } \\
\text { Phi }\end{array}$ \\
\hline Bus Selector1 & Alpha;Beta,qbar & off & $\begin{array}{l}\text { Alpha;Beta, Alpha Beta } \\
\text { qbar }\end{array}$ \\
\hline
\end{tabular}

Table 75. Constant Block Properties

\begin{tabular}{|l|l|l|l|l|l|l|l|}
\hline Name & Value & Sampling Mode & Out Min & Out Max & Out Data Type Str & Sample Time & Frame Period \\
\hline Airbrake demand & 0.0 & Sample based & {[]} & {[]} & $\begin{array}{l}\text { Inherit: Inherit from } \\
\text { 'Constant value' }\end{array}$ & inf \\
\hline qbar0 & qbar0 & Sample based & {[]} & {[]} & $\begin{array}{l}\text { Inherit: Inherit from } \\
\text { 'Constant value' }\end{array}$ & inf \\
\hline
\end{tabular}

Table 76. Demux Block Properties

\begin{tabular}{|c|c|c|c|}
\hline Name & Outputs & Display Option & Bus Selection Mode \\
\hline Demux1 & 3 & none & off \\
\hline
\end{tabular}

Table 77. Inport Block Properties

\begin{tabular}{|l|l|l|l|l|l|l|l|}
\hline Name & Port & Defined In Blk & Out Min & Out Max \\
\hline Airdata System o/p & 1 & Incidence, Sideslip, 1/2rhoV^2 & {[]} & {[]} & {[]} & {[]} \\
\hline Guidance o/p & 3 & Limit Demands & {[]} & {[]} \\
\hline IMU\&GPS o/p & 2 & p,q,r, Select Phi & {[]} \\
\hline
\end{tabular}

Table 78. Outport Block Properties

\begin{tabular}{|c|c|c|c|c|c|c|c|c|c|c|c|}
\hline Name & Port & $\begin{array}{l}\text { Icon } \\
\text { Display }\end{array}$ & Bus Object & $\begin{array}{l}\text { Var Size } \\
\text { Sig }\end{array}$ & $\begin{array}{l}\text { Out } \\
\text { Min }\end{array}$ & $\begin{array}{l}\text { Out } \\
\text { Max }\end{array}$ & $\begin{array}{l}\text { Out Data } \\
\text { Type Str }\end{array}$ & \begin{tabular}{|l|} 
Source Of \\
Initial \\
Output \\
Value
\end{tabular} & $\begin{array}{l}\text { Output } \\
\text { When } \\
\text { Disabled }\end{array}$ & $\begin{array}{l}\text { Initial } \\
\text { Output }\end{array}$ & Used By Blk \\
\hline Demands & 1 & $\begin{array}{l}\text { Port } \\
\text { number }\end{array}$ & BusObject & Inherit & [] & [] & $\begin{array}{l}\text { Inherit: } \\
\text { auto }\end{array}$ & Dialog & held & [] & Aero2Act \\
\hline
\end{tabular}

Table 79. PreLookup Block Properties

\begin{tabular}{|c|c|c|c|c|c|c|c|c|c|c|c|c|}
\hline Name & $\begin{array}{l}\text { Breakpoints } \\
\text { Data }\end{array}$ & $\begin{array}{l}\text { Index } \\
\text { Search } \\
\text { Method }\end{array}$ & $\begin{array}{l}\text { Begin } \\
\text { Index } \\
\text { Search } \\
\text { Using } \\
\text { Previous } \\
\text { Index } \\
\text { Result }\end{array}$ & $\begin{array}{l}\text { Output } \\
\text { Only } \\
\text { The } \\
\text { Index }\end{array}$ & $\begin{array}{l}\text { Process Out } \\
\text { Of Range } \\
\text { Input }\end{array}$ & $\begin{array}{l}\text { Use Last } \\
\text { Breakpoint }\end{array}$ & $\begin{array}{l}\text { Action } \\
\text { For } \\
\text { Out } \\
\text { Of } \\
\text { Range } \\
\text { Input }\end{array}$ & $\begin{array}{l}\text { Breakpoint } \\
\text { Min }\end{array}$ & $\begin{array}{l}\text { Breakpoint } \\
\text { Max }\end{array}$ & $\begin{array}{l}\text { Breakpoint } \\
\text { Data } \\
\text { Str }\end{array}$ & $\begin{array}{l}\text { Index } \\
\text { Data } \\
\text { Type } \\
\text { Str }\end{array}$ & $\begin{array}{l}\text { Fraction } \\
\text { Data } \\
\text { Type Str }\end{array}$ \\
\hline $\begin{array}{l}\text { Alpha } \\
\text { Schedule }\end{array}$ & a_vec & $\begin{array}{l}\text { Binary } \\
\text { search }\end{array}$ & on & off & $\begin{array}{l}\text { Linear } \\
\text { extrapolation }\end{array}$ & off & None & [] & [] & $\begin{array}{l}\text { Inherit: } \\
\text { Same } \\
\text { input }\end{array}$ & uint32 & $\begin{array}{l}\text { Inherit: } \\
\text { Inherit } \\
\text { via } \\
\text { internal } \\
\text { rule }\end{array}$ \\
\hline $\begin{array}{l}\text { Beta } \\
\text { Schedule }\end{array}$ & b_vec & $\begin{array}{l}\text { Binary } \\
\text { search }\end{array}$ & on & off & $\begin{array}{l}\text { Linear } \\
\text { extrapolation }\end{array}$ & off & None & [] & [] & $\begin{array}{l}\text { Inherit: } \\
\text { Same } \\
\text { input }\end{array}$ & uint32 & $\begin{array}{l}\text { Inherit: } \\
\text { Inherit } \\
\text { via } \\
\text { internal } \\
\text { rule }\end{array}$ \\
\hline
\end{tabular}


Take Off And Landing Stage Of Aircraft with Matlab Simulation

Table 80. Product Block Properties

\begin{tabular}{|l|l|l|l|l|l|l|l|l|}
\hline Name & Inputs & Multiplication & Collapse Mode & Collapse Dim & $\begin{array}{l}\text { Input } \\
\text { Same DT }\end{array}$ & $\begin{array}{l}\text { Out } \\
\text { Min }\end{array}$ & Out Max & Out Data Type Str \\
\hline Product1 & $* /$ & Element-wise(.*) & All dimensions & 1 & on & {[]} & {[]} & $\begin{array}{l}\text { Inherit: Same as first } \\
\text { input }\end{array}$ \\
\hline
\end{tabular}

Table 81. Selector Block Properties

\begin{tabular}{|c|c|c|c|c|c|c|c|c|c|}
\hline Name & $\begin{array}{l}\text { Number Of } \\
\text { Dimensions }\end{array}$ & $\begin{array}{l}\text { Index } \\
\text { Mode }\end{array}$ & $\begin{array}{ll}\text { Index } & \text { Option } \\
\text { Array } & \end{array}$ & $\begin{array}{l}\text { Index } \\
\text { Param } \\
\text { Array }\end{array}$ & $\begin{array}{l}\text { Output } \\
\text { Size Array }\end{array}$ & $\begin{array}{l}\text { Input Port } \\
\text { Width }\end{array}$ & Index Options & Indices & $\begin{array}{l}\text { Output } \\
\text { Sizes }\end{array}$ \\
\hline Alpha & 1 & $\begin{array}{l}\text { One- } \\
\text { based }\end{array}$ & $\begin{array}{l}\text { Index vector } \\
(\text { dialog) }\end{array}$ & 1 & 1 & 2 & $\begin{array}{ll}\text { Index } & \text { vector } \\
(\text { dialog) } & \end{array}$ & 1 & 1 \\
\hline Beta & 1 & $\begin{array}{l}\text { One- } \\
\text { based }\end{array}$ & $\begin{array}{l}\text { Index vector } \\
\text { (dialog) }\end{array}$ & 2 & 1 & 2 & $\begin{array}{ll}\text { Index } & \text { vector } \\
(\text { dialog) } & \end{array}$ & 2 & 1 \\
\hline
\end{tabular}

Table 82. Sum Block Properties

\begin{tabular}{|c|c|c|c|c|c|c|c|c|c|}
\hline Name & Icon Shape & Inputs & Collapse Mode & Collapse Dim & $\begin{array}{l}\text { Input Same } \\
\text { DT }\end{array}$ & $\begin{array}{l}\text { Accum Data Type } \\
\text { Str }\end{array}$ & $\begin{array}{l}\text { Out } \\
\text { Min }\end{array}$ & $\begin{array}{l}\text { Out } \\
\text { Max }\end{array}$ & $\begin{array}{l}\text { Out Data } \\
\text { Type Str }\end{array}$ \\
\hline Sum1 & round & ++1 & All dimensions & 1 & on & $\begin{array}{l}\text { Inherit: Inherit via } \\
\text { internal rule }\end{array}$ & [] & [] & $\begin{array}{l}\text { Inherit: Same } \\
\text { as first input }\end{array}$ \\
\hline Sum2 & round & ++1 & All dimensions & 1 & on & $\begin{array}{l}\text { Inherit: Inherit via } \\
\text { internal rule }\end{array}$ & [] & [] & $\begin{array}{l}\text { Inherit: Same } \\
\text { as first input }\end{array}$ \\
\hline Sum3 & round & ++1 & All dimensions & 1 & on & $\begin{array}{l}\text { Inherit: Inherit via } \\
\text { internal rule }\end{array}$ & [] & [] & $\begin{array}{l}\text { Inherit: Same } \\
\text { as first input }\end{array}$ \\
\hline
\end{tabular}

System - landing/Control System/Configurable Subsystem for 3 Controller Designs

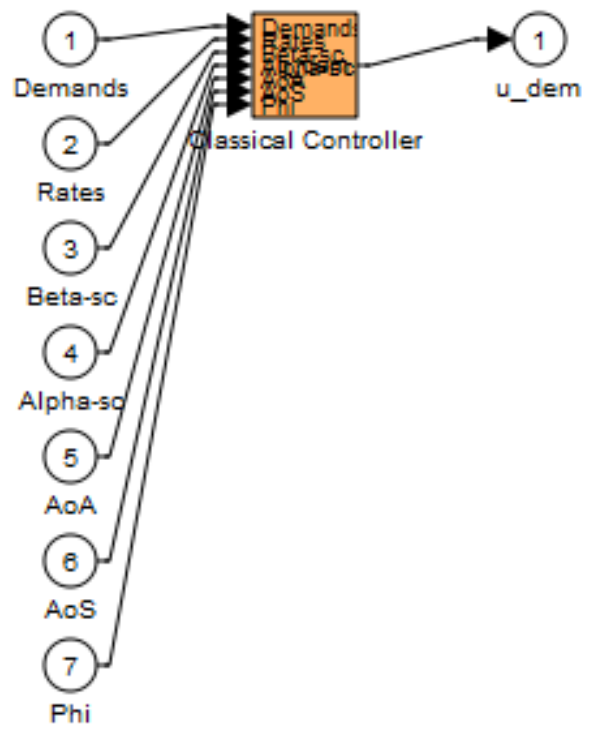

Table 83. Inport Block Properties

\begin{tabular}{|l|l|l|l|l|}
\hline Name & Port & Defined In Blk & Out Min & Out Max \\
\hline Alpha-sc & 4 & Alpha Schedule, $\underline{\text { Alpha Schedule }}$ & {[]} & {[]} \\
\hline AoA & 5 & Alpha & {[]} & {[]} \\
\hline AoS & 6 & $\underline{\text { Beta }}$ & {[]} & {[]} \\
\hline Beta-sc & 3 & Beta Schedule, Beta Schedule & {[]} & {[]} \\
\hline Demands & 1 & Limit Demands & {[]} & {[]} \\
\hline Phi & 7 & Select Phi & {[]} & {[]} \\
\hline Rates & 2 & p,q,r & {[]} & {[]} \\
\hline
\end{tabular}


Take Off And Landing Stage Of Aircraft with Matlab Simulation

Table 84. Outport Block Properties

\begin{tabular}{|l|l|l|l|l|l|l|l|l|l|l|l|}
\hline Name & Port & $\begin{array}{l}\text { Icon } \\
\text { Display }\end{array}$ & Bus Object & $\begin{array}{l}\text { Var Size } \\
\text { Sig }\end{array}$ & $\begin{array}{l}\text { Out } \\
\text { Min }\end{array}$ & $\begin{array}{l}\text { Out } \\
\text { Max }\end{array}$ & $\begin{array}{l}\text { Out Dource Of } \\
\text { Type Str }\end{array}$ & $\begin{array}{l}\text { Initial } \\
\text { Output } \\
\text { Value }\end{array}$ & $\begin{array}{l}\text { Output When } \\
\text { Disabled }\end{array}$ \\
\hline u_dem & 1 & $\begin{array}{l}\text { Port } \\
\text { number }\end{array}$ & BusObject & Inherit & {[]} & {[]} & $\begin{array}{l}\text { Inherit: } \\
\text { auto }\end{array}$ & Dialog & held & Dsed By Blk \\
\hline
\end{tabular}

System - landing/Control System/Trim Map

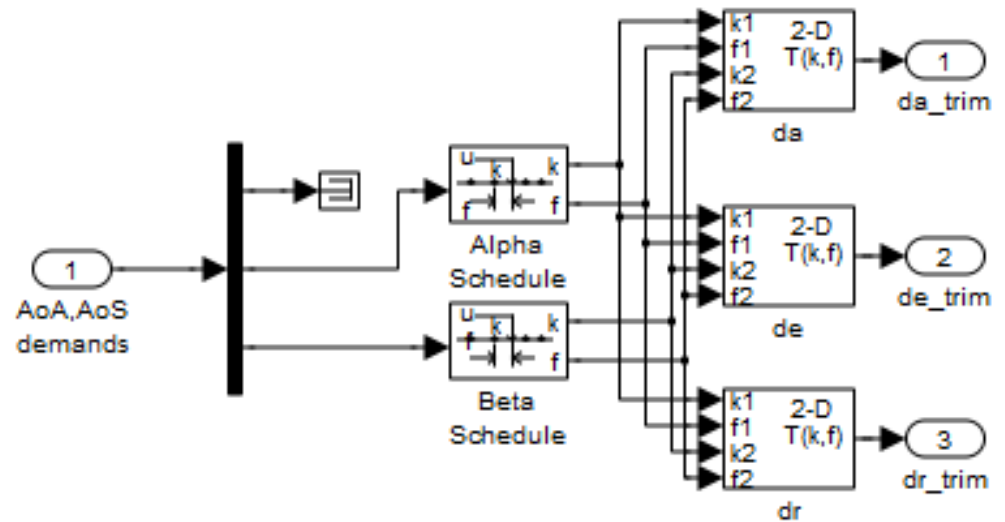

Table 85. Demux Block Properties

\begin{tabular}{|l|l|l|l|}
\hline Name & Outputs & Display Option & Bus Selection Mode \\
\hline Demux & 3 & none & off \\
\hline
\end{tabular}

Table 86. Inport Block Properties

\begin{tabular}{|l|l|l|l|l|}
\hline Name & Port & Defined In Blk & Out Min & Out Max \\
\hline AoA,AoS demands & 1 & $\underline{\text { Limit Demands }}$ & {[]} & {[]} \\
\hline
\end{tabular}

Table 87. Interpolation_n-D Block Properties

\begin{tabular}{|c|c|c|c|c|c|c|c|c|c|c|c|c|c|c|c|}
\hline Name & $\begin{array}{l}\text { Number } \\
\text { Of Table } \\
\text { Dimensi } \\
\text { ons }\end{array}$ & Table & $\begin{array}{l}\text { Inter } \\
\text { p } \\
\text { Meth } \\
\text { od }\end{array}$ & $\begin{array}{l}\text { Extra } \\
\text { p } \\
\text { Meth } \\
\text { od }\end{array}$ & \begin{tabular}{|l} 
Ran \\
ge \\
Erro \\
r \\
Mod \\
e
\end{tabular} & $\begin{array}{l}\text { Che } \\
\text { ck } \\
\text { Inde } \\
x \quad \text { In } \\
\text { Cod } \\
\text { e }\end{array}$ & $\begin{array}{l}\text { Vali } \\
\text { d } \\
\text { Inde } \\
\text { x } \\
\text { May } \\
\text { Reac } \\
\text { h } \\
\text { Last }\end{array}$ & $\begin{array}{l}\text { Num } \\
\text { Selecti } \\
\text { on } \\
\text { Dims }\end{array}$ & $\begin{array}{l}\text { Ou } \\
\mathbf{t} \\
\mathbf{M i} \\
\mathbf{n}\end{array}$ & $\begin{array}{l}\text { Ou } \\
\text { t } \\
\text { Ma } \\
\mathbf{x}\end{array}$ & $\begin{array}{l}\text { Out } \\
\text { Data } \\
\text { Type } \\
\text { Str }\end{array}$ & $\begin{array}{l}\text { Tab } \\
\text { le } \\
\text { Min }\end{array}$ & $\begin{array}{l}\text { Tab } \\
\text { le } \\
\text { Ma } \\
\mathbf{x}\end{array}$ & $\begin{array}{l}\text { Tabl } \\
\text { e } \\
\text { Data } \\
\text { Type } \\
\text { Str }\end{array}$ & $\begin{array}{l}\text { Intermedi } \\
\text { ate } \\
\text { Results } \\
\text { Data } \\
\text { Type Str }\end{array}$ \\
\hline da & 2 & $\begin{array}{l}\text { squeeze(U_t } \\
\text { rim_matrix ( } \\
1,:,:))\end{array}$ & Linear & Linear & $\begin{array}{l}\text { Non } \\
\mathrm{e}\end{array}$ & off & off & 0 & [] & [] & $\begin{array}{l}\text { Inher } \\
\text { it: } \\
\text { Inher } \\
\text { it } \\
\text { from } \\
\text { 'Tabl } \\
\text { e } \\
\text { data' }\end{array}$ & [] & [] & $\begin{array}{l}\text { Inher } \\
\text { it: } \\
\text { Same } \\
\text { as } \\
\text { outpu } \\
t\end{array}$ & $\begin{array}{l}\text { Inherit: } \\
\text { Same as } \\
\text { output }\end{array}$ \\
\hline de & 2 & $\begin{array}{l}\text { squeeze(U_t } \\
\text { rim_matrix ( } \\
2,:,:))\end{array}$ & Linear & Linear & $\begin{array}{l}\text { Non } \\
\text { e }\end{array}$ & off & off & 0 & [] & [] & $\begin{array}{l}\text { Inher } \\
\text { it: } \\
\text { Inher } \\
\text { it } \\
\text { from } \\
\text { 'Tabl } \\
\text { e } \\
\text { data' }\end{array}$ & [] & [] & $\begin{array}{l}\text { Inher } \\
\text { it: } \\
\text { Same } \\
\text { as } \\
\text { outpu } \\
t\end{array}$ & $\begin{array}{l}\text { Inherit: } \\
\text { Same as } \\
\text { output }\end{array}$ \\
\hline
\end{tabular}




\begin{tabular}{|c|c|c|c|c|c|c|c|c|c|c|c|c|c|c|c|}
\hline Name & $\begin{array}{l}\text { Number } \\
\text { Of Table } \\
\text { Dimensi } \\
\text { ons }\end{array}$ & Table & $\begin{array}{l}\text { Inter } \\
\text { p } \\
\text { Meth } \\
\text { od }\end{array}$ & $\begin{array}{l}\text { Extra } \\
\text { p } \\
\text { Meth } \\
\text { od }\end{array}$ & $\begin{array}{l}\text { Ran } \\
\text { ge } \\
\text { Erro } \\
\text { r } \\
\text { Mod } \\
\text { e }\end{array}$ & $\begin{array}{l}\text { Che } \\
\text { ck } \\
\text { Inde } \\
\text { x In } \\
\text { Cod } \\
\text { e }\end{array}$ & \begin{tabular}{|l} 
Vali \\
d \\
Inde \\
x \\
May \\
Reac \\
h \\
Last
\end{tabular} & $\begin{array}{l}\text { Num } \\
\text { Selecti } \\
\text { on } \\
\text { Dims } \\
\end{array}$ & $\begin{array}{l}\text { Ou } \\
\text { t } \\
\text { Mi } \\
\text { n }\end{array}$ & $\begin{array}{l}\text { Ou } \\
\text { t } \\
\text { Ma } \\
\mathbf{x}\end{array}$ & \begin{tabular}{|l|} 
Out \\
Data \\
Type \\
Str
\end{tabular} & $\begin{array}{l}\text { Tab } \\
\text { le } \\
\text { Min }\end{array}$ & \begin{tabular}{|l} 
Tab \\
le \\
Ma \\
$\mathbf{x}$
\end{tabular} & $\begin{array}{l}\text { Tabl } \\
\text { e } \\
\text { Data } \\
\text { Type } \\
\text { Str }\end{array}$ & $\begin{array}{l}\text { Intermedi } \\
\text { ate } \\
\text { Results } \\
\text { Data } \\
\text { Type Str }\end{array}$ \\
\hline $\mathrm{dr}$ & 2 & $\begin{array}{l}\text { squeeze(U_t } \\
\text { rim_matrix( } \\
3,:,:))\end{array}$ & Linear & Linear & $\begin{array}{l}\text { Non } \\
\mathrm{e}\end{array}$ & off & off & 0 & [] & [] & $\begin{array}{l}\text { Inher } \\
\text { it: } \\
\text { Inher } \\
\text { it } \\
\text { from } \\
\text { 'Tabl } \\
\text { e } \\
\text { data' }\end{array}$ & [] & [] & $\begin{array}{l}\text { Inher } \\
\text { it: } \\
\text { Same } \\
\text { as } \\
\text { outpu } \\
\text { t }\end{array}$ & $\begin{array}{l}\text { Inherit: } \\
\text { Same as } \\
\text { output }\end{array}$ \\
\hline
\end{tabular}

Table 88. Outport Block Properties

\begin{tabular}{|l|l|l|l|l|l|l|l|l|l|l|l|l|}
\hline Name & Port & $\begin{array}{l}\text { Icon } \\
\text { Display }\end{array}$ & Bus Object & $\begin{array}{l}\text { Var Size } \\
\text { Sig }\end{array}$ & $\begin{array}{l}\text { Out } \\
\text { Min }\end{array}$ & $\begin{array}{l}\text { Out } \\
\text { Max }\end{array}$ & $\begin{array}{l}\text { Out } \\
\text { Type Str }\end{array}$ & $\begin{array}{l}\text { Source } \\
\text { Initial } \\
\text { Output } \\
\text { Value }\end{array}$ & $\begin{array}{l}\text { Output When } \\
\text { Disabled }\end{array}$ & $\begin{array}{l}\text { Initial } \\
\text { Output }\end{array}$ & $\begin{array}{l}\text { Used By } \\
\text { Blk }\end{array}$ \\
\hline da_trim & 1 & Port number & BusObject & Inherit & {[]} & {[]} & $\begin{array}{l}\text { Inherit: } \\
\text { auto }\end{array}$ & Dialog & held & Sum2 \\
\hline de_trim & 2 & Port number & BusObject & Inherit & {[]} & {[]} & $\begin{array}{l}\text { Inherit: } \\
\text { auto }\end{array}$ & Dialog & held & Sum1 \\
\hline dr_trim & 3 & Port number & BusObject & Inherit & {[]} & {[]} & $\begin{array}{l}\text { Inherit: } \\
\text { auto }\end{array}$ & Dialog & held & Sum3 \\
\hline
\end{tabular}

Table 89. PreLookup Block Properties

\begin{tabular}{|c|c|c|c|c|c|c|c|c|c|c|c|c|}
\hline Name & $\begin{array}{l}\text { Breakpoin } \\
\text { ts Data }\end{array}$ & $\begin{array}{l}\text { Index } \\
\text { Search } \\
\text { Method }\end{array}$ & \begin{tabular}{|l} 
Begin \\
Index \\
Search \\
Using \\
Previous \\
Index \\
Result
\end{tabular} & $\begin{array}{l}\text { Output } \\
\text { Only } \\
\text { The } \\
\text { Index }\end{array}$ & $\begin{array}{l}\text { Process Out } \\
\text { Of Range } \\
\text { Input }\end{array}$ & $\begin{array}{l}\text { Use Last } \\
\text { Breakpoint }\end{array}$ & \begin{tabular}{|l|} 
Action \\
For \\
Out \\
Of \\
Range \\
Input
\end{tabular} & $\begin{array}{l}\text { Breakpo } \\
\text { int Min }\end{array}$ & $\begin{array}{l}\text { Breakpoi } \\
\text { nt Max }\end{array}$ & $\begin{array}{l}\text { Breakpoint } \\
\text { Data Type } \\
\text { Str }\end{array}$ & $\begin{array}{l}\text { Index } \\
\text { Data } \\
\text { Type } \\
\text { Str }\end{array}$ & $\begin{array}{l}\text { Fraction } \\
\text { Data } \\
\text { Type Str }\end{array}$ \\
\hline $\begin{array}{l}\text { Alpha } \\
\text { Schedule }\end{array}$ & a_vec & $\begin{array}{l}\text { Binary } \\
\text { search }\end{array}$ & on & off & $\begin{array}{l}\text { Linear } \\
\text { extrapolation }\end{array}$ & off & None & [] & [] & $\begin{array}{l}\text { Inherit: } \\
\text { Same } \\
\text { input }\end{array}$ & uint32 & $\begin{array}{l}\text { Inherit: } \\
\text { Inherit } \\
\text { via } \\
\text { internal } \\
\text { rule }\end{array}$ \\
\hline $\begin{array}{l}\text { Beta } \\
\text { Schedule }\end{array}$ & b_vec & $\begin{array}{l}\text { Binary } \\
\text { search }\end{array}$ & on & off & $\begin{array}{l}\text { Linear } \\
\text { extrapolation }\end{array}$ & off & None & [] & [] & $\begin{array}{l}\text { Inherit: } \\
\text { Same } \\
\text { input }\end{array}$ & uint32 & $\begin{array}{l}\text { Inherit: } \\
\text { Inherit } \\
\text { via } \\
\text { internal } \\
\text { rule }\end{array}$ \\
\hline
\end{tabular}

Table 90. Terminator Block Properties

\begin{tabular}{|l|}
\hline Name \\
\hline Terminator \\
\hline
\end{tabular}




\section{System - $\underline{\text { landing/Scopes }}$}

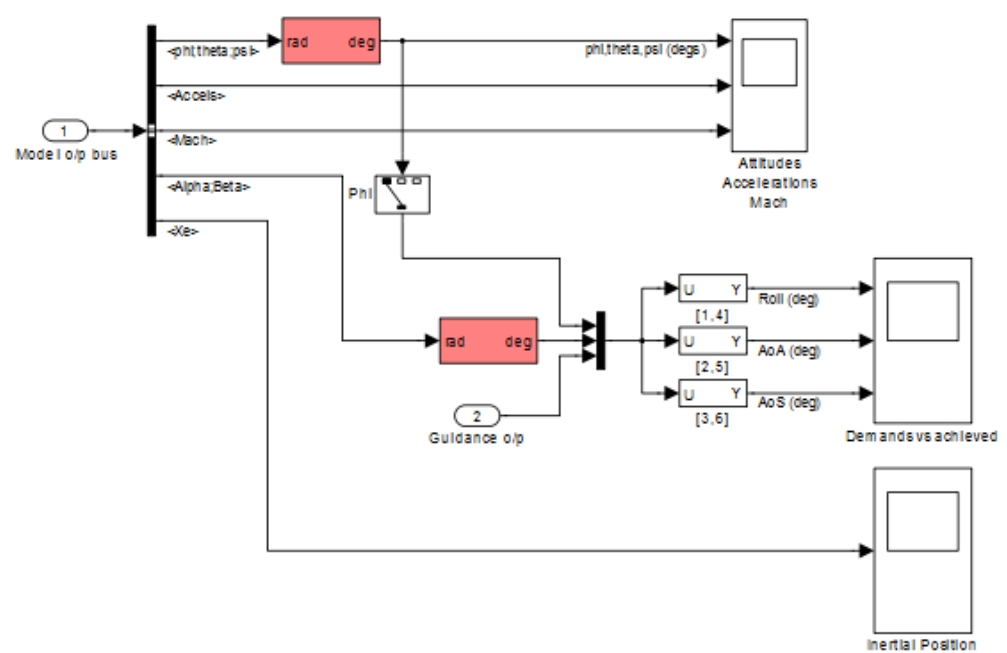

Table 91. Angle Conversion Block Properties

\begin{tabular}{|l|l|l|}
\hline Name & IU & OU \\
\hline Angle Conversion & rad & deg \\
\hline Angle Conversion3 & rad & deg \\
\hline
\end{tabular}

Table 92. BusSelector Block Properties

\begin{tabular}{|l|l|l|l|}
\hline Name & Output Signals & Output As Bus & Input Signals \\
\hline & & & pdot;qdot;rdot \\
& & & pqr \\
Bus Selector & phi;theta;psi,Accels,Mach,Alpha;Beta,Xe & off & phi;theta;psi \\
& & & DCM \\
& & Accels \\
& & & Vb \\
& & & Ve \\
& & & Xe \\
& & & Alpha;Beta, Alpha Beta $\}$ \\
\hline
\end{tabular}

Table 93. Inport Block Properties

\begin{tabular}{|l|l|l|l|l|}
\hline Name & Port & Defined In Blk & $\begin{array}{l}\text { Out } \\
\text { Min }\end{array}$ & Out Max \\
\hline Guidance o/p & 2 & $\underline{\text { Limit Demands }}$ & {[]} \\
\hline Model o/p bus & 1 & $\begin{array}{l}\text { Reshape, p,q,r, phi theta psi, Reshape (9) to [3x3] column-major, Force ---> Acc, Unit } \\
\text { Conversion, Unit Conversion, xe,ye,ze, Incidence, Sideslip, Product1, 1/2rhoV^2 }\end{array}$ & {[]} \\
\hline
\end{tabular}

Table 94. Mux Block Properties

\begin{tabular}{|l|l|l|}
\hline Name & Inputs & Display Option \\
\hline Mux & {$[1,2,3]$} & bar \\
\hline
\end{tabular}

Table 95. Selector Block Properties

\begin{tabular}{|c|c|c|c|c|c|c|c|c|c|c|c|}
\hline Name & $\begin{array}{l}\text { Number } \\
\text { Dimensions }\end{array}$ & Of & $\begin{array}{l}\text { Index } \\
\text { Mode }\end{array}$ & $\begin{array}{l}\text { Index Option } \\
\text { Array }\end{array}$ & $\begin{array}{l}\text { Index } \\
\text { Param } \\
\text { Array }\end{array}$ & $\begin{array}{l}\text { Output } \\
\text { Size Array }\end{array}$ & $\begin{array}{l}\text { Input } \\
\text { Width }\end{array}$ & Port & Index Options & Indices & Output Sizes \\
\hline$[1,4]$ & 1 & & One-based & $\begin{array}{l}\text { Index vectos } \\
\text { (dialog) }\end{array}$ & {$\left[\begin{array}{ll}1 & 4\end{array}\right]$} & 1 & 6 & & $\begin{array}{ll}\text { Index } & \text { vector } \\
(\text { dialog }) & \end{array}$ & {$\left[\begin{array}{ll}1 & 4\end{array}\right]$} & 1 \\
\hline$[2,5]$ & 1 & & One-based & $\begin{array}{l}\text { Index vectos } \\
(\text { dialog) }\end{array}$ & [ $\left.\begin{array}{ll}2 & 5\end{array}\right]$ & 1 & 6 & & $\begin{array}{ll}\text { Index } & \text { vector } \\
(\text { dialog }) & \end{array}$ & [2 5] & 1 \\
\hline
\end{tabular}


Take Off And Landing Stage Of Aircraft with Matlab Simulation

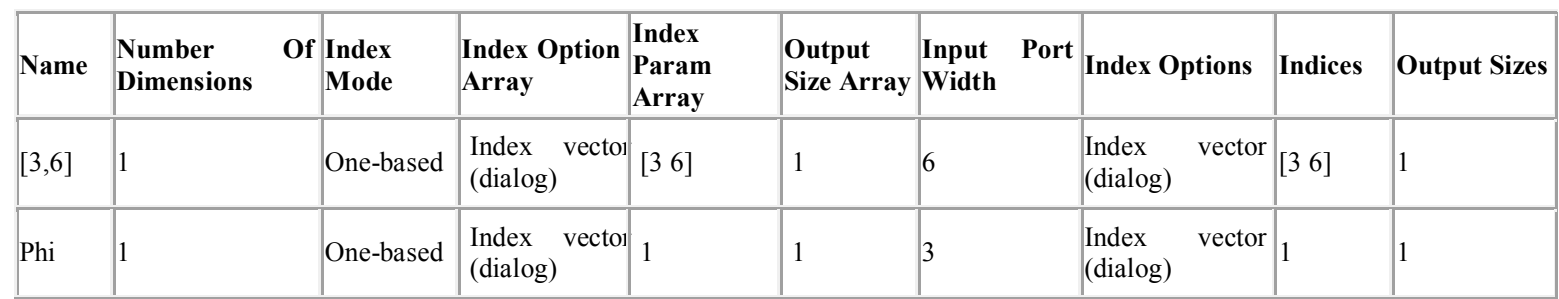

System - landing/Visualization

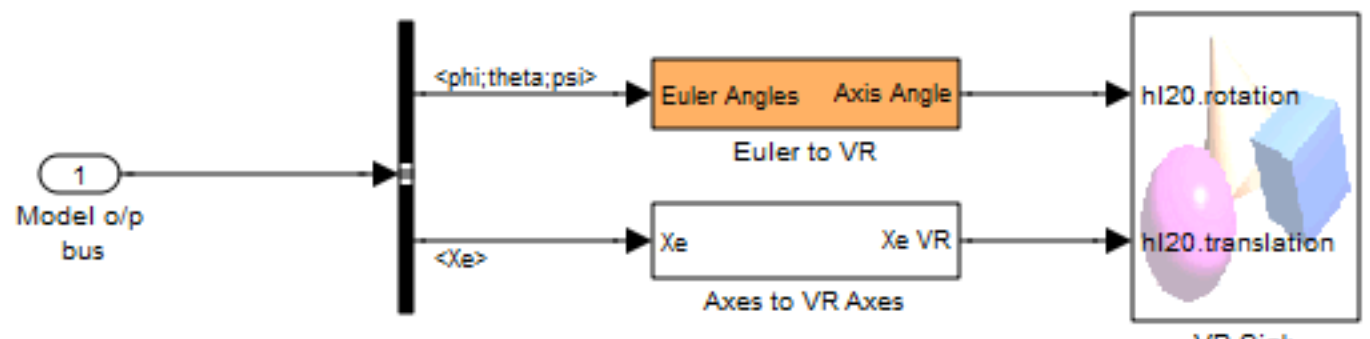

VR Sink

Table 96. BusSelector Block Properties

\begin{tabular}{|l|l|l|l|}
\hline Name & Output Signals & Output As Bus & Input Signals \\
\hline & & & pdot;qdot;rdot \\
& & & pqr \\
Bus Selector & phi; theta;psi,Xe & phi; & DCM \\
& & & Accels \\
& & Vb \\
& & Ve \\
& & Xe \\
& & $\{$ Alpha;Beta, Alpha Beta $\}$ \\
& & Mach \\
\hline
\end{tabular}

Table 97. Inport Block Properties

\begin{tabular}{|ll|l|l|l|l|}
\hline Name & Port & Defined In Blk & $\begin{array}{l}\text { Out } \\
\text { Min }\end{array}$ & Out Max \\
\hline $\begin{array}{llllll}\text { Model } \\
\text { bus }\end{array}$ & o/p & 1 & $\begin{array}{l}\text { Reshape, p,q,r, phi theta psi, Reshape (9) to [3x3] column-major, Force ---> Acc, Unit } \\
\text { Conversion, Unit Conversion, xe,ye,ze, Incidence, Sideslip, Product1, 1/2rhoV^2 }\end{array}$ & {$\left[\begin{array}{ll}\text { [] } \\
\hline\end{array}\right.$} \\
\hline
\end{tabular}

Table 98. Virtual Reality Sink Block Properties

\begin{tabular}{|l|l|l|l|l|l|l|l|l|}
\hline Name & $\begin{array}{l}\text { Sample } \\
\text { Time }\end{array}$ & $\begin{array}{l}\text { View } \\
\text { Enable }\end{array}$ & $\begin{array}{l}\text { Remote } \\
\text { View }\end{array}$ & Fields Written & World File Name & $\begin{array}{l}\text { World } \\
\text { Description }\end{array}$ & $\begin{array}{l}\text { Auto } \\
\text { View }\end{array}$ & $\begin{array}{l}\text { Video } \\
\text { Dimensions }\end{array}$ \\
\hline $\begin{array}{l}\text { VR } \\
\text { Sink }\end{array}$ & 0.1 & on & off & hl20.rotation.4\#hl20.translation.3 & aeroblk_HL20.wrl & aeroblk_HL20 & on & {[]} \\
\hline
\end{tabular}

System - landing/isualization/Axes to VR Axes

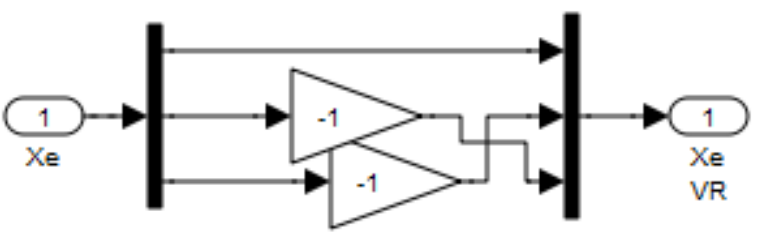


Table 99. Demux Block Properties

\begin{tabular}{|l|l|l|l|}
\hline Name & Outputs & Display Option & Bus Selection Mode \\
\hline Demux & 3 & none & off \\
\hline
\end{tabular}

Table 100. Gain Block Properties

\begin{tabular}{|c|c|c|c|c|c|c|c|c|c|}
\hline Name & Gain & Multiplication & Param Min & Param Max & $\begin{array}{l}\text { Param } \\
\text { Str }\end{array}$ & Data Type & Out Min & Out Max & Out Data Type Str \\
\hline rad-->deg & -1 & Element-wise $(\mathrm{K} . * \mathrm{u})$ & [] & [] & $\begin{array}{l}\text { Inherit: } \\
\text { input }\end{array}$ & Same as & [] & [] & $\begin{array}{l}\text { Inherit: Same as } \\
\text { input }\end{array}$ \\
\hline rad-->deg1 & -1 & Element-wise $\left(\mathrm{K} . *^{*} \mathrm{u}\right)$ & [] & [] & $\begin{array}{l}\text { Inherit: } \\
\text { input }\end{array}$ & Same as & [] & [] & $\begin{array}{l}\text { Inherit: Same as } \\
\text { input }\end{array}$ \\
\hline
\end{tabular}

Table 101. Inport Block Properties

\begin{tabular}{|l|l|l|l|l|}
\hline Name & Port & Defined In Blk & Out Min & Out Max \\
\hline $\mathrm{Xe}$ & 1 & xe,ye,ze & {[]} & {[]} \\
\hline
\end{tabular}

Table 102. Mux Block Properties

\begin{tabular}{|l|l|l|}
\hline Name & Inputs & Display Option \\
\hline Mux & 3 & bar \\
\hline
\end{tabular}

Table 103. Outport Block Properties

\begin{tabular}{|c|c|c|c|c|c|c|c|c|c|c|c|}
\hline Name & Port & $\begin{array}{l}\text { Icon } \\
\text { Display }\end{array}$ & Bus Object & $\begin{array}{ll}\text { Var } & \text { Size } \\
\text { Sig } & \end{array}$ & $\begin{array}{l}\text { Out } \\
\text { Min }\end{array}$ & $\begin{array}{l}\text { Out } \\
\text { Max }\end{array}$ & $\begin{array}{l}\text { Out Data } \\
\text { Type Str }\end{array}$ & $\begin{array}{l}\text { Source Of } \\
\text { Initial } \\
\text { Output Value }\end{array}$ & $\begin{array}{l}\text { Output When } \\
\text { Disabled }\end{array}$ & $\begin{array}{l}\text { Initial } \\
\text { Output }\end{array}$ & $\begin{array}{l}\text { Used By } \\
\text { Blk }\end{array}$ \\
\hline Xe VR & 1 & Port number & BusObject & Inherit & [] & [] & Inherit: auto & Dialog & held & [] & VR Sink \\
\hline
\end{tabular}

System - landing/isualization/Euler to VR

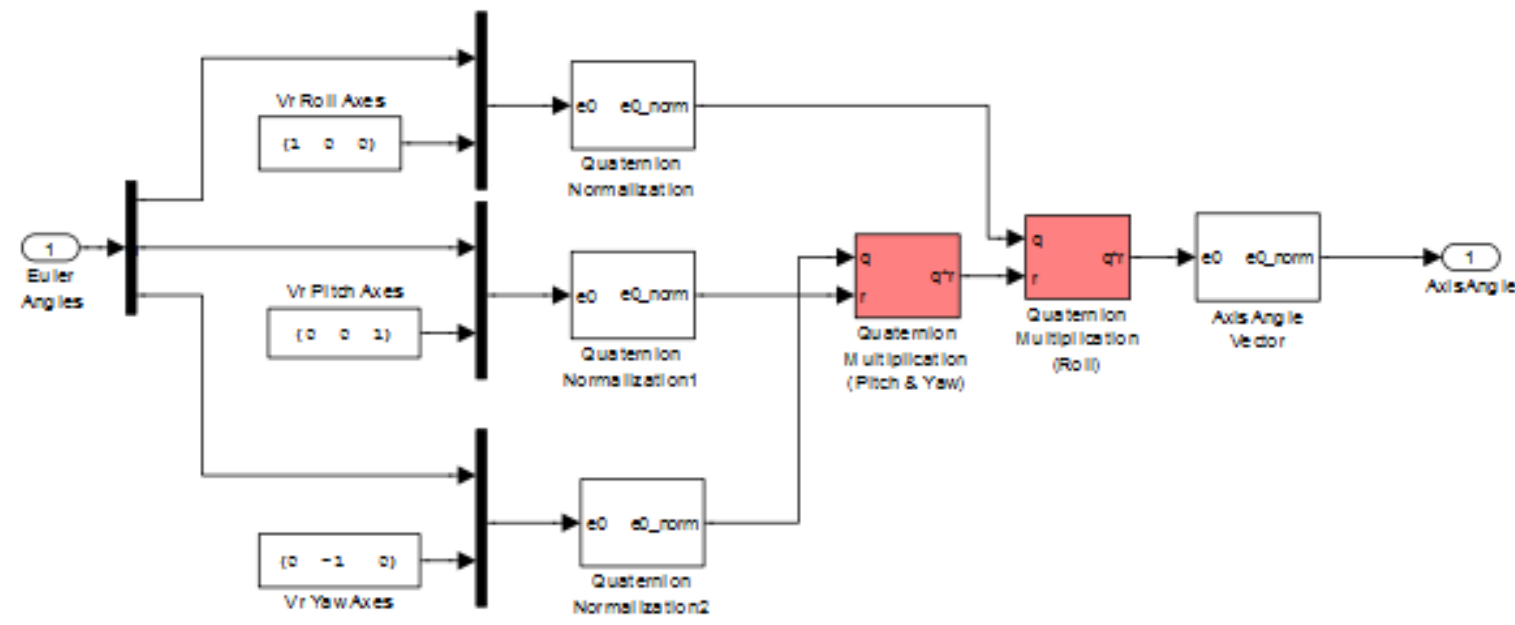

Table 104. Constant Block Properties

\begin{tabular}{|l|l|l|l|l|l|l|l|}
\hline Name & Value & Sampling Mode & $\begin{array}{l}\text { Out } \\
\text { Min }\end{array}$ & $\begin{array}{l}\text { Out } \\
\text { Max }\end{array}$ & Out Data Type Str & Sample Time & Frame Period \\
\hline Vr Pitch Axes & {$\left[\begin{array}{lll}0 & 0 & 1\end{array}\right]$} & Sample based & {[]} & {[]} & Inherit: Inherit from 'Constant value' & inf & inf \\
\hline Vr Roll Axes & {$\left[\begin{array}{lll}1 & 0 & 0\end{array}\right]$} & Sample based & {[]} & {[]} & Inherit: Inherit from 'Constant value' & inf \\
\hline Vr Yaw Axes & {$\left[\begin{array}{lll}0 & -1 & 0\end{array}\right]$} & Sample based & {[]} & {[]} & Inherit: Inherit from 'Constant value' & inf \\
\hline
\end{tabular}

Table 105. Demux Block Properties

\begin{tabular}{|l|l|l|l|}
\hline Name & Outputs & Display Option & Bus Selection Mode \\
\hline Demux & 3 & none & off \\
\hline
\end{tabular}


Table 106. Inport Block Properties

\begin{tabular}{|l|l|l|l|l|}
\hline Name & Port & Defined In Blk & Out Min & Out Max \\
\hline Euler Angles & 1 & phi theta psi & {[]} & {[]} \\
\hline
\end{tabular}

Table 107. Mux Block Properties

\begin{tabular}{|l|l|l|}
\hline Name & Inputs & Display Option \\
\hline Mux & 2 & bar \\
\hline Mux1 & 2 & bar \\
\hline Mux2 & 2 & bar \\
\hline
\end{tabular}

Table 108. Outport Block Properties

\begin{tabular}{|c|c|c|c|c|c|c|c|c|c|c|c|}
\hline Name & Port & $\begin{array}{l}\text { Icon } \\
\text { Display }\end{array}$ & Bus Object & $\begin{array}{ll}\text { Var } & \text { Size } \\
\text { Sig } & \end{array}$ & $\begin{array}{l}\text { Out } \\
\text { Min }\end{array}$ & $\begin{array}{l}\text { Out } \\
\text { Max }\end{array}$ & $\begin{array}{l}\text { Out Data } \\
\text { Type Str }\end{array}$ & $\begin{array}{l}\text { Source Of } \\
\text { Initial } \\
\text { Output } \\
\text { Value }\end{array}$ & $\begin{array}{l}\text { Output When } \\
\text { Disabled }\end{array}$ & $\begin{array}{l}\text { Initial } \\
\text { Output }\end{array}$ & $\begin{array}{l}\text { Used By } \\
\text { Blk }\end{array}$ \\
\hline $\begin{array}{l}\text { Axis } \\
\text { Angle }\end{array}$ & 1 & Port number & BusObject & Inherit & [] & [] & Inherit: auto & Dialog & held & [] & VR Sink \\
\hline
\end{tabular}

Table 109. Quaternion Multiplication Block Properties

\begin{tabular}{|l|}
\hline Name \\
\hline Quaternion Multiplication (Pitch \& Yaw) \\
\hline Quaternion Multiplication (Roll) \\
\hline
\end{tabular}

System - landing/Visualization/Euler to VR/Axis Angle Vector

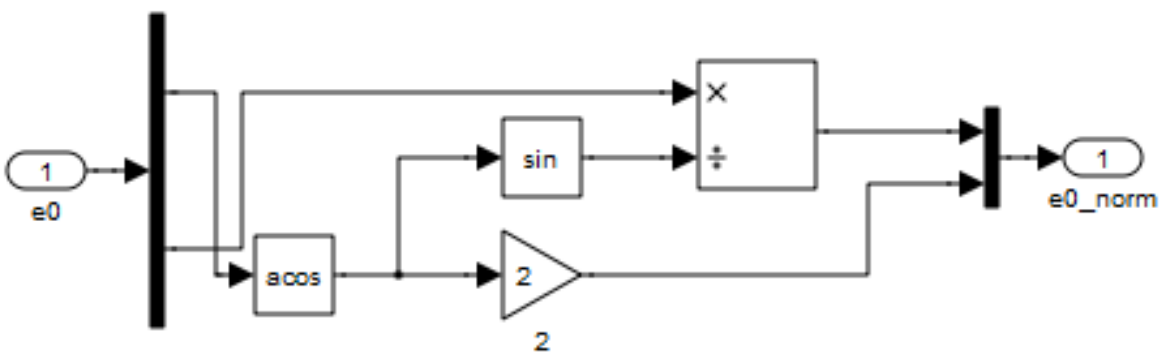

Table 110. Demux Block Properties

\begin{tabular}{|l|l|l|l|}
\hline Name & Outputs & Display Option & Bus Selection Mode \\
\hline Demux & {$\left[\begin{array}{lll}1 & 3\end{array}\right]$} & none & off \\
\hline
\end{tabular}

Table 111. Gain Block Properties

\begin{tabular}{|l|l|l|l|l|l|l|l|l|}
\hline Name & Gain & Multiplication & Param Min & Param Max & $\begin{array}{l}\text { Param Data Type } \\
\text { Str }\end{array}$ & Out Min & Out Max & Out Data Type Str \\
\hline 2 & 2 & Element-wise(K.*u) & {[]} & {[]} & Inherit: Same as input & {[]} & {[]} & $\begin{array}{l}\text { Inherit: } \\
\text { input }\end{array}$ \\
\hline
\end{tabular}

Table 112. Inport Block Properties

\begin{tabular}{|l|l|l|l|l|}
\hline Name & Port & Defined In Blk & Out Min & Out Max \\
\hline $\mathrm{e} 0$ & 1 & Sum, Sum, Sum, Sum & {[]} & {[]} \\
\hline
\end{tabular}

Table 113. Mux Block Properties

\begin{tabular}{|l|l|l|}
\hline Name & Inputs & Display Option \\
\hline Mux & 2 & bar \\
\hline
\end{tabular}


Take Off And Landing Stage Of Aircraft with Matlab Simulation

Table 114. Outport Block Properties

\begin{tabular}{|c|c|c|c|c|c|c|c|c|c|c|c|}
\hline Name & $\begin{array}{l}\text { Por } \\
t\end{array}$ & $\begin{array}{l}\text { Icon } \\
\text { Display }\end{array}$ & Bus Object & $\begin{array}{ll}\text { Var } & \text { Size } \\
\text { Sig } & \end{array}$ & $\begin{array}{l}\text { Out } \\
\text { Min }\end{array}$ & $\begin{array}{l}\text { Out } \\
\text { Max }\end{array}$ & $\begin{array}{l}\text { Out Data } \\
\text { Type Str }\end{array}$ & \begin{tabular}{|l|} 
Source \\
Initial \\
Output \\
Value
\end{tabular} & $\begin{array}{l}\text { Output When } \\
\text { Disabled }\end{array}$ & $\begin{array}{l}\text { Initial } \\
\text { Output }\end{array}$ & $\begin{array}{l}\text { Used } \\
\text { By Blk }\end{array}$ \\
\hline e0_norm & 1 & Port number & BusObject & Inherit & [] & [] & $\begin{array}{l}\text { Inherit: } \\
\text { auto }\end{array}$ & Dialog & held & [] & $\frac{\mathrm{VR}}{\text { Sink }}$ \\
\hline
\end{tabular}

Table 115. Product Block Properties

\begin{tabular}{|l|l|l|l|l|l|l|l|l|}
\hline Name & Inputs & Multiplication & Collapse Mode & Collapse Dim & $\begin{array}{l}\text { Input Same } \\
\text { DT }\end{array}$ & Out Min & Out Max & Out Data Type Str \\
\hline Product & $* /$ & Element-wise(.*) & All dimensions & 1 & on & {[]} & {[]} & $\begin{array}{l}\text { Inherit: Same as first } \\
\text { input }\end{array}$ \\
\hline
\end{tabular}

Table 116. Trigonometry Block Properties

\begin{tabular}{|l|l|l|l|l|}
\hline Name & Operator & Approximation Method & Number Of Iterations & Output Signal Type \\
\hline Trigonometric Function & $\sin$ & None & 11 & auto \\
\hline Trigonometric Function2 & acos & None & 11 & auto \\
\hline
\end{tabular}

System - landing/Visualization/Euler to VR/Quaternion Normalization

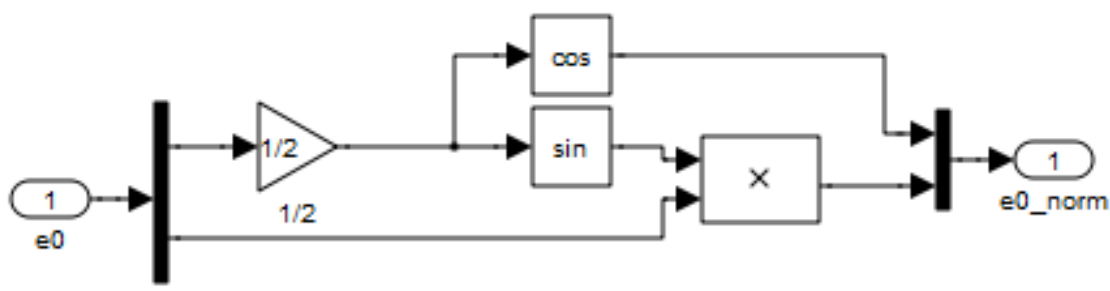

Table 117. Demux Block Properties

\begin{tabular}{|l|l|l|l|}
\hline Name & Outputs & Display Option & Bus Selection Mode \\
\hline Demux & {$\left[\begin{array}{lll}1 & 3\end{array}\right]$} & none & off \\
\hline
\end{tabular}

Table 118. Gain Block Properties

\begin{tabular}{|l|l|l|l|l|l|l|l|l|l|}
\hline Name & Gain & Multiplication & Param Min & Param Max & $\begin{array}{l}\text { Param Data Type } \\
\text { Str }\end{array}$ & Out Min & Out Max & Out Data Type Str \\
\hline $1 / 2$ & $1 / 2$ & Element-wise(K.*u) & {[]} & {[]} & Inherit: Same as input & {[]} & {[]} & $\begin{array}{l}\text { Inherit: } \\
\text { input }\end{array}$ \\
\hline
\end{tabular}

Table 119. Inport Block Properties

\begin{tabular}{|l|l|l|l|l|}
\hline Name & Port & Defined In Blk & Out Min & Out Max \\
\hline $\mathrm{e} 0$ & 1 & $\underline{\text { Demux }}, \underline{\text { Vr Roll Axes }}$ & {[]} & {[]} \\
\hline
\end{tabular}

Table 120. Mux Block Properties

\begin{tabular}{|l|l|l|}
\hline Name & Inputs & Display Option \\
\hline Mux & 2 & bar \\
\hline
\end{tabular}

Table 121. Outport Block Properties

\begin{tabular}{|c|c|c|c|c|c|c|c|c|c|c|c|c|}
\hline Name & Port & $\begin{array}{l}\text { Icon } \\
\text { Display }\end{array}$ & Bus Object & $\begin{array}{l}\text { Var Size } \\
\text { Sig }\end{array}$ & $\begin{array}{l}\text { Out } \\
\text { Min }\end{array}$ & $\begin{array}{l}\text { Out } \\
\text { Max }\end{array}$ & $\begin{array}{l}\text { Out Data } \\
\text { Type Str }\end{array}$ & $\begin{array}{l}\text { Source Of } \\
\text { Initial } \\
\text { Output } \\
\text { Value }\end{array}$ & $\begin{array}{l}\text { Output } \\
\text { When } \\
\text { Disabled }\end{array}$ & $\begin{array}{l}\text { Initial } \\
\text { Output }\end{array}$ & $\begin{array}{l}\text { Used } \\
\text { Blk }\end{array}$ & By \\
\hline e0_norm & 1 & $\begin{array}{l}\text { Port } \\
\text { number }\end{array}$ & BusObject & Inherit & [] & [] & $\begin{array}{l}\text { Inherit: } \\
\text { auto }\end{array}$ & Dialog & held & [] & $\begin{array}{l}\text { Demux, } \\
\text { Demux, } \\
\text { Demux, } \\
\text { Demux }\end{array}$ & \\
\hline
\end{tabular}


Take Off And Landing Stage Of Aircraft with Matlab Simulation

Table 122. Product Block Properties

\begin{tabular}{|l|l|l|l|l|l|l|l|l|}
\hline Name & Inputs & Multiplication & Collapse Mode & Collapse Dim & $\begin{array}{l}\text { Input } \\
\text { Same DT }\end{array}$ & Out Min & Out Max & Out Data Type Str \\
\hline Product & 2 & Element-wise(*) & All dimensions & 1 & on & {[]} & {[]} & $\begin{array}{l}\text { Inherit: Same as first } \\
\text { input }\end{array}$ \\
\hline
\end{tabular}

Table 123. Trigonometry Block Properties

\begin{tabular}{|l|l|l|l|l|}
\hline Name & Operator & Approximation Method & Number Of Iterations & Output Signal Type \\
\hline Trigonometric Function & $\sin$ & None & 11 & auto \\
\hline Trigonometric Function 1 & $\cos$ & None & 11 & auto \\
\hline
\end{tabular}

System - landing/Visualization/Euler to VR/Quaternion Normalization1

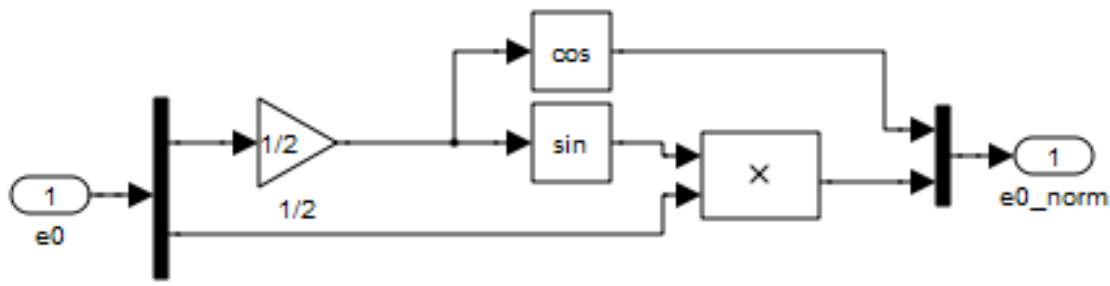

Table 124. Demux Block Properties

\begin{tabular}{|l|l|l|l|}
\hline Name & Outputs & Display Option & Bus Selection Mode \\
\hline Demux & {$\left[\begin{array}{lll}1 & 3\end{array}\right]$} & none & off \\
\hline
\end{tabular}

Table 125. Gain Block Properties

\begin{tabular}{|l|l|l|l|l|l|l|l|l|}
\hline Name & Gain & Multiplication & Param Min & Param Max & $\begin{array}{l}\text { Param Data Type } \\
\text { Str }\end{array}$ & Out Min & Out Max & Out Data Type Str \\
\hline $1 / 2$ & $1 / 2$ & Element-wise(K.*u) & {[]} & {[]} & Inherit: Same as input & {[]} & {[]} & $\begin{array}{l}\text { Inherit: } \\
\text { input }\end{array}$ \\
\hline
\end{tabular}

Table 126. Inport Block Properties

\begin{tabular}{|l|l|l|l|l|}
\hline Name & Port & Defined In Blk & Out Min & Out Max \\
\hline $\mathrm{e} 0$ & 1 & $\underline{\text { Demux }}, \underline{\text { Vr Pitch Axes }}$ & {[]} & {[]} \\
\hline
\end{tabular}

Table 127. Mux Block Properties

\begin{tabular}{|l|l|l|}
\hline Name & Inputs & Display Option \\
\hline Mux & 2 & bar \\
\hline
\end{tabular}

Table 128. Outport Block Properties

\begin{tabular}{|c|c|c|c|c|c|c|c|c|c|c|c|}
\hline Name & Port & $\begin{array}{l}\text { Icon } \\
\text { Display }\end{array}$ & Bus Object & $\begin{array}{l}\text { Var Size } \\
\text { Sig }\end{array}$ & $\begin{array}{l}\text { Out } \\
\text { Min }\end{array}$ & $\begin{array}{l}\text { Out } \\
\text { Max }\end{array}$ & $\begin{array}{l}\text { Out Data } \\
\text { Type Str }\end{array}$ & \begin{tabular}{|l|} 
Source Of \\
Initial \\
Output \\
Value
\end{tabular} & $\begin{array}{l}\text { Output } \\
\text { When } \\
\text { Disabled }\end{array}$ & $\begin{array}{l}\text { Initial } \\
\text { Output }\end{array}$ & Used By Blk \\
\hline e0_norm & 1 & $\begin{array}{l}\text { Port } \\
\text { number }\end{array}$ & BusObject & Inherit & [] & [] & $\begin{array}{l}\text { Inherit: } \\
\text { auto }\end{array}$ & Dialog & held & [] & $\begin{array}{l}\text { Demux1, } \\
\text { Demux1, } \\
\text { Demux1, } \\
\text { Demux1 }\end{array}$ \\
\hline
\end{tabular}

Table 129. Product Block Properties

\begin{tabular}{|c|c|c|c|c|c|c|c|c|}
\hline Name & Inputs & Multiplication & Collapse Mode & Collapse Dim & $\begin{array}{l}\text { Input } \\
\text { Same DT }\end{array}$ & Out Min & Out Max & Out Data Type Str \\
\hline Product & 2 & Element-wise $\left(.^{*}\right)$ & All dimensions & 1 & on & [] & [] & $\begin{array}{l}\text { Inherit: Same as first } \\
\text { input }\end{array}$ \\
\hline
\end{tabular}


Table 130. Trigonometry Block Properties

\begin{tabular}{|l|l|l|l|l|}
\hline Name & Operator & Approximation Method & Number Of Iterations & Output Signal Type \\
\hline Trigonometric Function & $\sin$ & None & 11 & auto \\
\hline Trigonometric Function1 & $\cos$ & None & 11 & auto \\
\hline
\end{tabular}

\section{System - landing/Visualization/Euler to VR/Quaternion Normalization2}

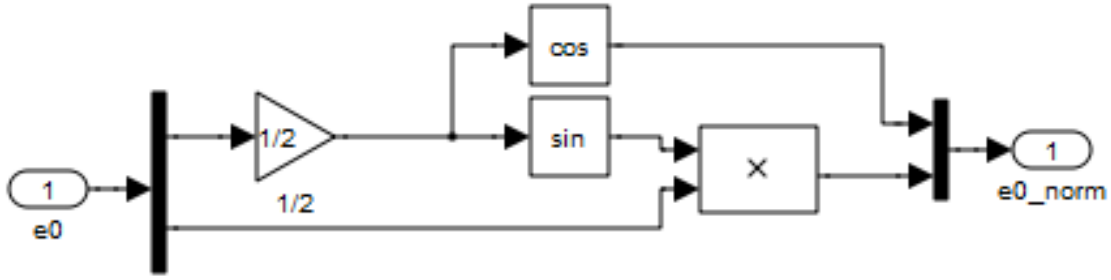

Table 131. Demux Block Properties

\begin{tabular}{|l|l|l|l|}
\hline Name & Outputs & Display Option & Bus Selection Mode \\
\hline Demux & {$\left[\begin{array}{lll}1 & 3\end{array}\right]$} & none & off \\
\hline
\end{tabular}

Table 132. Gain Block Properties

\begin{tabular}{|c|c|c|c|c|c|c|c|c|}
\hline Name & Gain & Multiplication & Param Min & Param Max & \begin{tabular}{|l|} 
Param \\
Type Str
\end{tabular} & Out Min & Out Max & $\begin{array}{l}\text { Out Data Type } \\
\text { Str }\end{array}$ \\
\hline $1 / 2$ & $1 / 2$ & Element-wise $\left(\mathrm{K} .{ }^{*} \mathrm{u}\right)$ & [] & [] & $\begin{array}{l}\text { Inherit: Same as } \\
\text { input }\end{array}$ & [] & [] & $\begin{array}{l}\text { Inherit: Same as } \\
\text { input }\end{array}$ \\
\hline
\end{tabular}

Table 133. Inport Block Properties

\begin{tabular}{|c|c|c|c|c|}
\hline Name & Port & Defined In Blk & Out Min & Out Max \\
\hline $\mathrm{e} 0$ & 1 & Demux, $\underline{\text { Vr Yaw Axes }}$ & [] & {[]} \\
\hline
\end{tabular}

Table 134. Mux Block Properties

\begin{tabular}{|l|l|l|}
\hline Name & Inputs & Display Option \\
\hline Mux & 2 & bar \\
\hline
\end{tabular}

Table 135. Outport Block Properties

\begin{tabular}{|c|c|c|c|c|c|c|c|c|c|c|c|}
\hline Name & Port & $\begin{array}{l}\text { Icon } \\
\text { Display }\end{array}$ & Bus Object & $\begin{array}{ll}\text { Var } & \text { Size } \\
\text { Sig } & \end{array}$ & $\begin{array}{l}\text { Out } \\
\text { Min }\end{array}$ & $\begin{array}{l}\text { Out } \\
\text { Max }\end{array}$ & $\begin{array}{l}\text { Out Data } \\
\text { Type Str }\end{array}$ & $\begin{array}{l}\text { Source Of } \\
\text { Initial } \\
\text { Output } \\
\text { Value }\end{array}$ & $\begin{array}{l}\text { Output } \\
\text { When } \\
\text { Disabled }\end{array}$ & $\begin{array}{l}\text { Initial } \\
\text { Output }\end{array}$ & $\begin{array}{l}\text { Used } \\
\text { Blk }\end{array}$ \\
\hline e0_norm & 1 & $\begin{array}{l}\text { Port } \\
\text { number }\end{array}$ & BusObject & Inherit & [] & [] & $\begin{array}{l}\text { Inherit: } \\
\text { auto }\end{array}$ & Dialog & held & [] & $\begin{array}{l}\text { Demux, } \\
\text { Demux, } \\
\text { Demux, } \\
\text { Demux }\end{array}$ \\
\hline
\end{tabular}

Table 136. Product Block Properties

\begin{tabular}{|l|l|l|l|l|l|l|l|l|}
\hline Name & Inputs & Multiplication & Collapse Mode & Collapse Dim & $\begin{array}{l}\text { Input Same } \\
\text { DT }\end{array}$ & Out Min Out Max & Out Data Type Str \\
\hline Product & 2 & Element-wise(*) & All dimensions & 1 & on & {[]} & {[]} \\
\hline
\end{tabular}

Table 137. Trigonometry Block Properties

\begin{tabular}{|l|l|l|l|l|}
\hline Name & Operator & Approximation Method & Number Of Iterations & Output Signal Type \\
\hline Trigonometric Function & $\sin$ & None & 11 & auto \\
\hline Trigonometric Function1 & $\cos$ & None & 11 & auto \\
\hline
\end{tabular}

\section{Appendix}


Table 138. Revision History

\begin{tabular}{|l|l|l|}
\hline Ver & Date & Description \\
\hline 1.0 & Wed Mar 13 13:42:21 2002 & \\
\hline
\end{tabular}

Table 139. Block Type Count

\begin{tabular}{|c|c|c|}
\hline BlockType & Count & Block Names \\
\hline Inport & 36 & 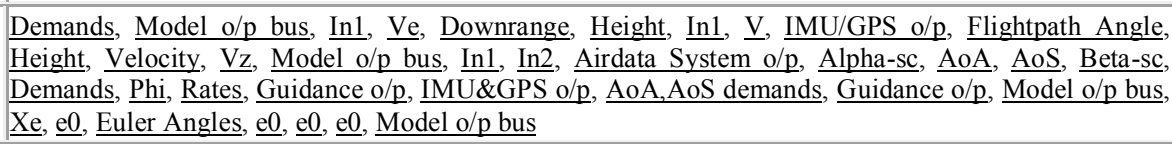 \\
\hline SubSystem & 24 & 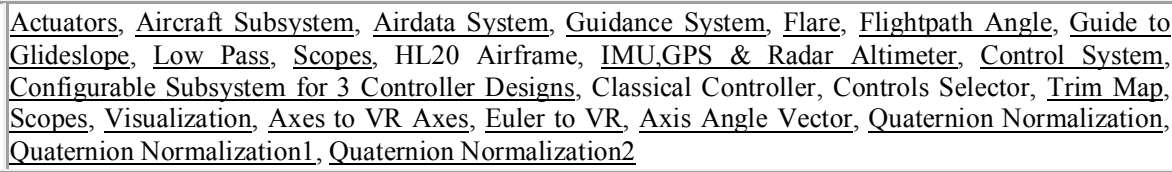 \\
\hline Outport & 24 & 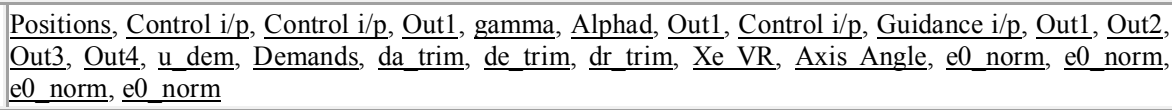 \\
\hline Gain & 13 & 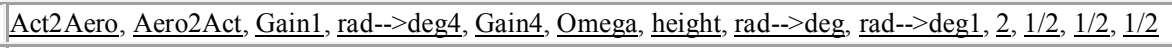 \\
\hline Selector & 10 & $\underline{\mathrm{u}, \mathrm{v}}, \underline{\mathrm{w}}, \underline{\text { Selector}}, \underline{\text { Select Phi }}, \underline{\text { Alpha }}, \underline{\text { Beta }}, \underline{\text { Phi }}, \underline{[1,4]}, \underline{[2,5]}, \underline{[3,6]}$ \\
\hline Mux & 10 & $\underline{M u x}, \underline{M u x}, \underline{M u x}, \underline{M u x}, \underline{M u x}, \underline{M u x 1}, \underline{M u x 2}, \underline{M u x}, \underline{M u x}, \underline{M u x}$ \\
\hline Demux & 10 & $\underline{\text { Demux }}, \underline{\text { Demux }}, \underline{\text { Demux1}}, \underline{\text { Demux }}, \underline{\text { Demux }}, \underline{\text { Demux }}, \underline{\text { Demux }}, \underline{\text { Demux }}, \underline{\text { Demux }}, \underline{\text { Demux }}$ \\
\hline Trigonometry & 9 & 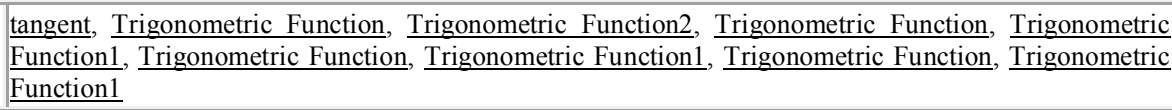 \\
\hline Constant & 9 & 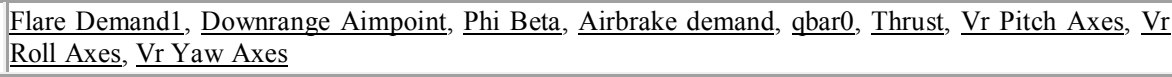 \\
\hline Sum & 8 & $\underline{\text { Sum2}}, \underline{\text { Sum2}}, \underline{\text { Sum } 3}, \underline{\text { Sum1}}, \underline{\text { Sum2}}, \underline{\text { Sum1 }}, \underline{\text { Sum2 }}, \underline{\text { Sum } 3}$ \\
\hline Product & 8 & product, product1 $, \underline{1 / \mathrm{V}}, \underline{\text { Product } 1}, \underline{\text { Product }}, \underline{\text { Product}}, \underline{\text { Product }}, \underline{\text { Product }}$ \\
\hline BusCreator & 8 & $\begin{array}{l}\text { Bus Creator1, Bus Creator1, Bus Creator, Bus Creator1, Model o/p bus, Bus Creator, Bus Creator1, Bus } \\
\text { Creator2 }\end{array}$ \\
\hline BusSelector & 7 & Bus Selector, Bus Selector, Bus Selector, Bus Selector, Bus Selector1, Bus Selector, Bus Selector \\
\hline Scope & 5 & $\begin{array}{l}\text { Guidance Performance, Limited Actuators, Attitudes Accelerations Mach, Demands vs achieved, } \\
\text { Inertial Position }\end{array}$ \\
\hline Angle Conversion (m) & 5 & Angle Conversion, Angle Conversion, Angle Conversion, Angle Conversion, Angle Conversion3 \\
\hline PreLookup & 4 & Alpha Schedule, Beta Schedule, $\underline{\text { Alpha Schedule, Beta Schedule }}$ \\
\hline Interpolation_n-D & 3 & $\underline{\mathrm{da}}, \underline{\mathrm{de}}, \underline{\mathrm{dr}}$ \\
\hline Integrator & 3 & Alphad1, Alpha Demand, Integrator \\
\hline Velocity Conversion (m) & 2 & V2, Velocity Conversion \\
\hline TransferFen & 2 & Phase Advance, Phase Advancel \\
\hline Terminator & 2 & Terminator, Terminator \\
\hline $\begin{array}{l}\text { Quaternion } \\
\text { Multiplication (m) }\end{array}$ & 2 & Quaternion Multiplication (Pitch \& Yaw), Quaternion Multiplication (Roll) \\
\hline Math & 2 & $\underline{\mathrm{v}}, \underline{\mathrm{V}}$ \\
\hline Virtual Reality Sink (m) & 1 & VR Sink \\
\hline UnitDelay & 1 & Unit Delay \\
\hline Switch & 1 & Flare Switch \\
\hline Stop & 1 & Stop Simulation \\
\hline $\begin{array}{l}\text { Second Order Nonlinear } \\
\text { Actuator }(\mathrm{m})\end{array}$ & 1 & Second Order Nonlinear Actuator \\
\hline Saturate & 1 & Limit Demands \\
\hline Lookup & 1 & Glideslope Trajectory \\
\hline Logic & 1 & Logical Operator \\
\hline Length Conversion (m) & 1 & Length Conversion \\
\hline Fen & 1 & $\underline{\mathrm{h}=0}$ \\
\hline EnablePort & 1 & Enable \\
\hline DotProduct & 1 & Dot Product \\
\hline $\begin{array}{l}\text { Compare To Constant } \\
\text { (m) }\end{array}$ & 1 & Flare Condition \\
\hline
\end{tabular}


Table 140. Model Variables

\begin{tabular}{|c|c|c|c|}
\hline Variable Name & Parent Blocks & Calling string & Value \\
\hline Act2Aero & Act2Aero & Act2Aero & $\left.\begin{array}{lllllllll}{[0.5} & -0.5 & 0 & 0 & 0 & 0 & 0 \\
0.5 & 0.5 & 0 & 0 & 0 & 0 & 0 & ; \\
0 & 0 & 0 & 0 & 0 & 0 & 1 & ; \\
0 & 0 & 0.5 & 0.5 & 0 & 0 & 0 ; \\
0 & 0 & 0 & 0 & 0.5 & 0.5 & 0 ; \\
0 & 0 & 0.5 & -0.5 & 0.5 & -0.5 & 0\end{array}\right]$ \\
\hline Aero2Act & Aero2Act & Aero2Act & 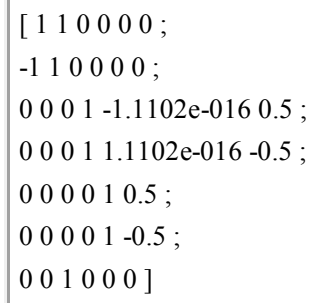 \\
\hline Aimpoint & Downrange Aimpoint & Aimpoint & 0 \\
\hline U_trim_matrix & $\begin{array}{l}\frac{\mathrm{da}}{\mathrm{de}} \\
\underline{\mathrm{dr}}\end{array}$ & $\begin{array}{l}\text { squeeze(U_trim_matrix }(1,: ;:)) \\
\text { squeeze(U_trim_matrix }(2,: ;:)) \\
\text { squeeze(U_trim_matrix }(3,:,:))\end{array}$ & {$[3 \times 8 \times 5$ double $]$} \\
\hline Xglide & Glideslope Trajectory & Xglide & [1x301 double] \\
\hline a_vec & $\begin{array}{l}\text { Alpha Schedule } \\
\text { Alpha Schedule }\end{array}$ & $\begin{array}{l}\text { a_vec } \\
\text { a_vec }\end{array}$ & {$\left[\begin{array}{llllllll}-9.9 & -5 & 0.01 & 5 & 10 & 15 & 20 & 25\end{array}\right]$} \\
\hline alpha0 & Alpha Demand & alpha $0 * 180 /$ pi & 0.1143 \\
\hline b_vec & $\frac{\text { Beta Schedule }}{\underline{\text { Beta Schedule }}}$ & $\begin{array}{l}\text { b_vec } \\
\text { b_vec }\end{array}$ & {$\left[\begin{array}{llllll}-9.9 & -5 & 0.01 & 5 & 9.9\end{array}\right]$} \\
\hline hglide & Glideslope Trajectory & hglide & [1 $1 \times 301$ double $]$ \\
\hline max_lim & $\begin{array}{l}\text { Second Order Nonlinear } \\
\text { Actuator }\end{array}$ & max_lim & {$\left[\begin{array}{llllllll}30 & 30 & 60 & 60 & 0 & 0 & 60\end{array}\right]$} \\
\hline min_lim & $\begin{array}{l}\text { Second Order Nonlinear } \\
\text { Actuator }\end{array}$ & min_lim & {$\left[\begin{array}{lllllll}-30 & -30 & 0 & 0 & -60 & -60 & -60\end{array}\right]$} \\
\hline qbar0 & qbar0 & qbar0 & $1.0723 \mathrm{e}+004$ \\
\hline wn_act & $\begin{array}{l}\text { Second Order Nonlinear } \\
\text { Actuator }\end{array}$ & wn_act & 44 \\
\hline z_act & $\begin{array}{l}\text { Second Order Nonlinear } \\
\text { Actuator }\end{array}$ & z_act & 0.7071 \\
\hline
\end{tabular}

Table 141. Model Functions

\begin{tabular}{|l|l|l|}
\hline Function Name & Parent Blocks & Calling string \\
\hline pi & $\underline{\text { Alpha Demand }}$ & alpha0*180/pi \\
\hline squeeze & $\underline{\mathrm{da}}$ & $\left.\begin{array}{l}\text { squeeze(U_trim_matrix }(1,:,:)) \\
\text { squeeze }\left(U_{-} \text {trim_matrix }(2,:,:)\right) \\
\text { squeeze }\left(U_{\text {de }} \text { trim_matrix }(3,:,:)\right)\end{array}\right)$ \\
\hline
\end{tabular}


IV. Result Of Simulation On Landing Stage

Height, Flightpath Angle, Velocity and Vertical Speed of Aircraf

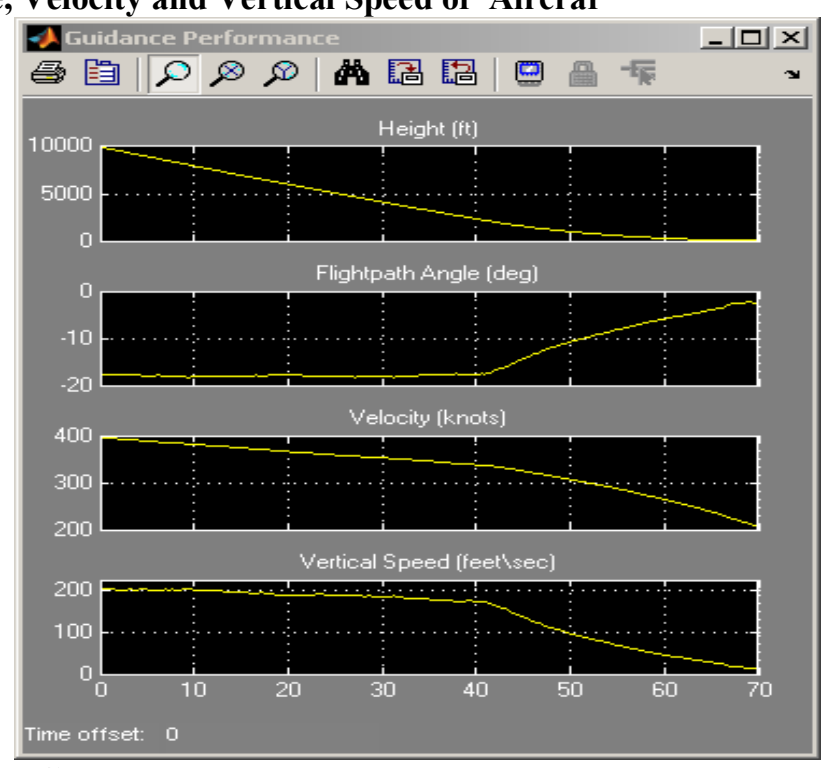

Demand and Achievement Graph

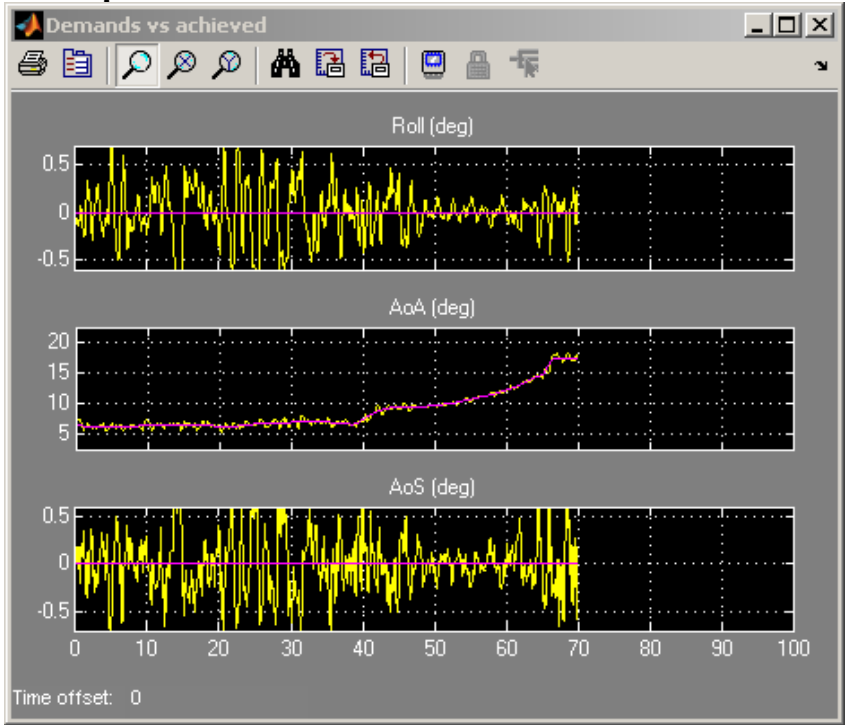

Attitudes Accelerations Mach

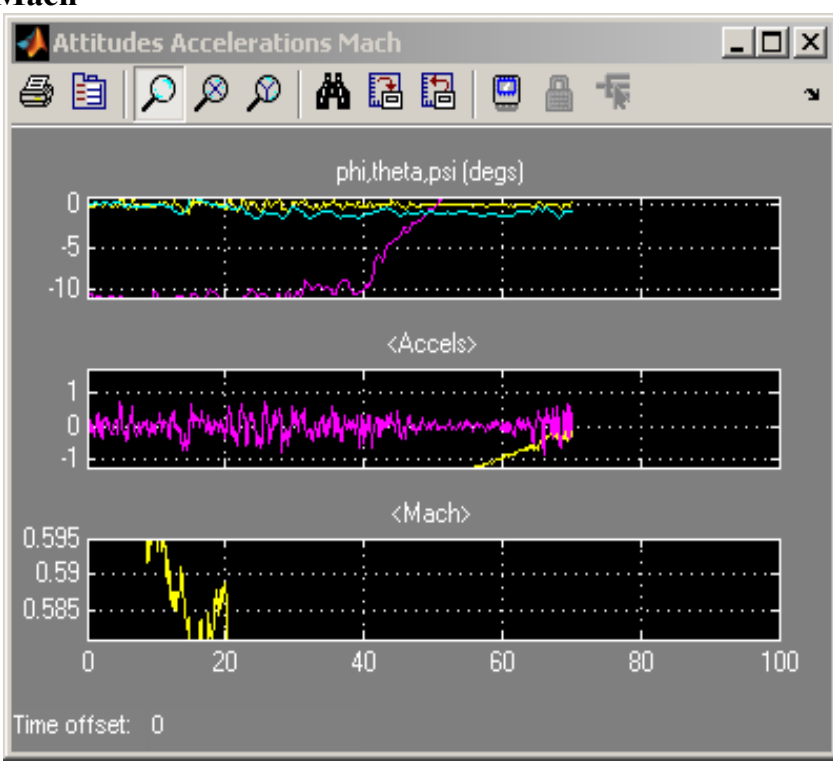




\section{Inertial Position}

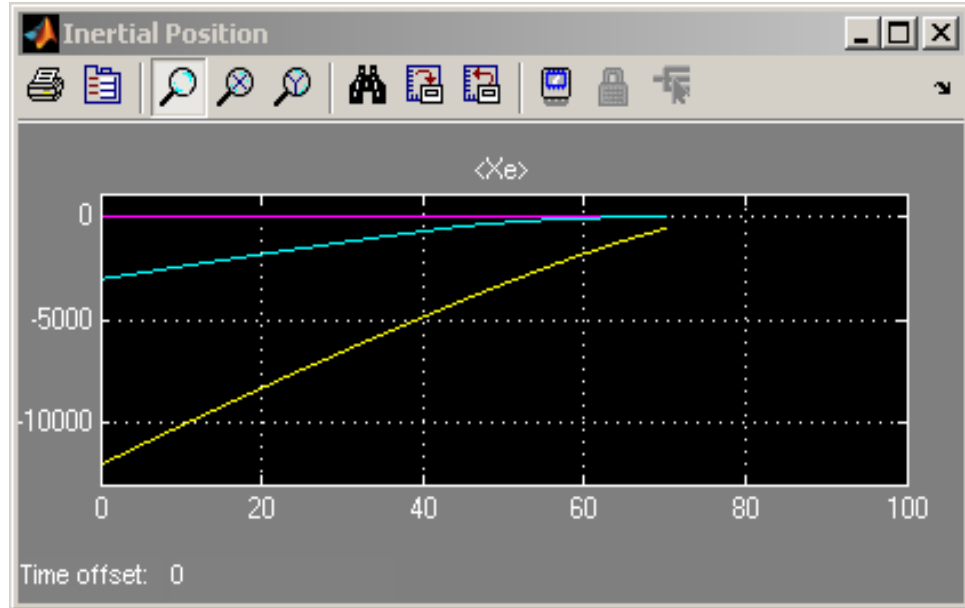

\section{Simulation Frame to Show Landing Stage of Aircraft}

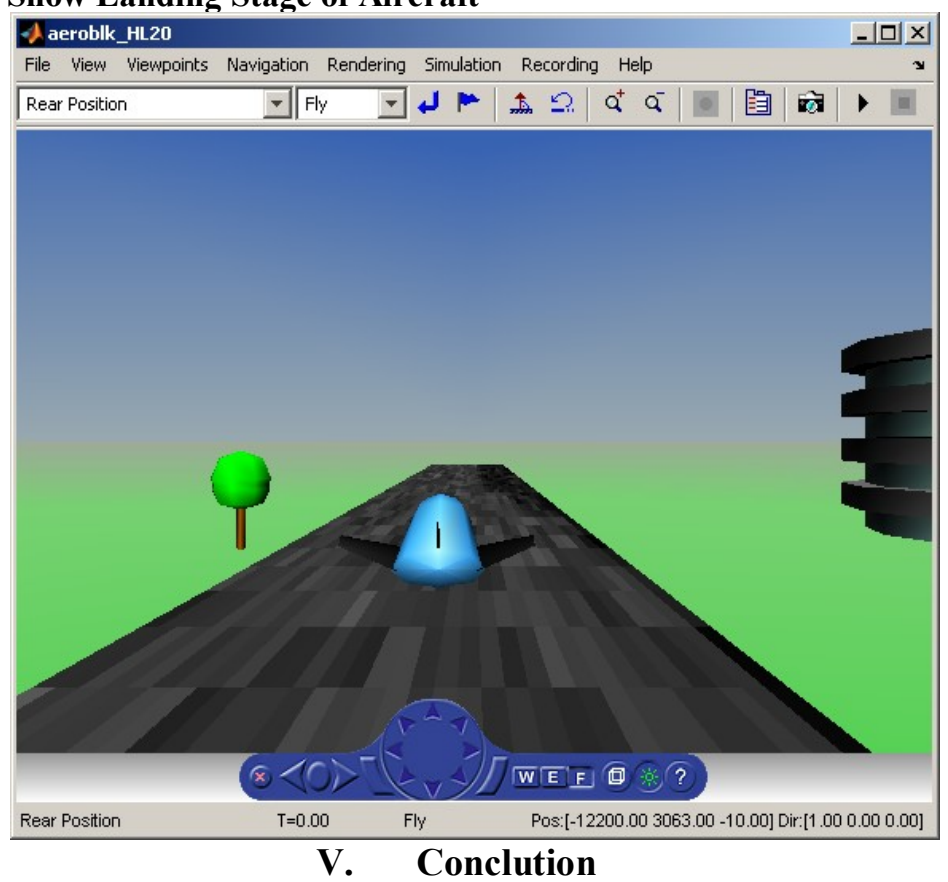

Atomic Subsystem. A subsystem treated as a unit by an implementation of the design documented in this report. The implementation computes the outputs of all the blocks in the atomic subsystem before computing the next block in the parent system's block execution order (sorted list).

Block Diagram. A Simulink block diagram represents a set of simultaneous equations that relate a system or subsystem's inputs to its outputs as a function of time. Each block in the diagram represents an equation of the form $y=f(t, x, u)$ where $t$ is the current time, $u$ is a block input, $y$ is a block output, and $x$ is a system state (see the Simulink documentation for information on the functions represented by the various types of blocks that make up the diagram). Lines connecting the blocks represent dependencies amonng the blocks, i.e., inputs whose current values are the outputs of other blocks. An implementation of a design described in this document computes a root or atomic system's outputs at each time step by computing the outputs of the blocks in an order determined by block input/output dependencies.

Block Parameter. A variable that determines the output of a block along with its inputs, for example, the gain parameter of a Gain block.

Block Execution Order. The order in which Simulink evaluates blocks during simulation of a model.The block execution order determined by Simulink ensures that a block executes only after all blocks on whose outputs it depends are executed.

Checksum. A number that indicates whether different versions of a model or atomic subsystem differ functionally or only cosmetically. Different checksums for different versions of the same model or subsystem indicate that the versions differ functionally. 
Design Variable. A symbolic (MATLAB) variable or expression used as the value of a block parameter.Design variables allow the behavior of the model to be altered by altering the value of the design variable.

Signal. A block output, so-called because block outputs typically vary with time.

Virtual Subsystem. A subsystem that is purely graphical, i.e., is intended to reduce the visual complexity of the block diagram of which it is a subsystem. An implementation of the design treats the blocks in the subsystem as part of the first nonvirtual ancestor of the virtual subsystem .

\section{References}

[1]. Visit site for take off and landing but more useful site is www.mathworks.com

[2]. Simulation with matlab programing

[3]. Matlab Programming by Kirani Singh

[4]. Matlab for Aero Space Engineering

[5]. Making Perfect Takeoff \& Landing in Light Airolanes by Ron Fowler

[6]. Advanced sliding mode control for mechanical systems Design, analysis and MATLAB simulation

[7]. Visualized Flight Maneuvers Handbook -High Wing

[8]. Practice Test Standards: CFI - Single-Engine FAA

[9]. Aircraft LandingGear Design Principle and Practices by Norman S. Currey

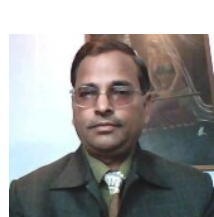

Biography

\section{Dr. A.K. Bhardwaj}

Dr. A.K. Bhardwaj is working as -Associate Professor\| in the Department of Electrical and Electronics Engineering, Faculty of Engineering and Technology of Sam Higginbottom Institute of Agriculture, Technology \& Sciences (Formerly AAI-DU) Allahabad, India from last 7 years after obtaining M. Tech. degree from Indian Institute of Technology Delhi, India in 2005. He has competed his Ph.D. degree from Sam Higginbottom Institute of Agriculture, Technology \& Sciences (Formerly AAI-DU) Allahabad, India in July 2010. Earlier he was -Assistant Professor\| in department of Electrical and Electronics Engineering, IMS Engineering College Ghaziabad (U.P.) India in the year 2005. He also worked for 6 years as faculty with IIT Ghaziabad (U.P.) India. $\mathrm{He}$ is also having practical experience with top class multinational companies during 1985-1998. His research interest includes, power management, energy management, reactive power control in electrical distribution system.

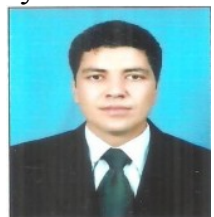

\section{Er. Naser.F.AB.EImajdub}

This Work Supported by Electric Engineering Department, Sam Higginbottom Institute of Agriculture, Technology \& Science Allahabad india Er. Naser.F.AB.Elmajdub — Phd student Btech from Technology College of Civil Aviation \& Meterology In year 1995 Tripoli -Libya M.tech from Sam Higginbottom Institute of Agriculture, Technology \& Science in year 2012 